\title{
SEISMIC ISOLATION SYSTEMS FOR REDUCING THE DYNAMIC RESPONSE OF THE ELEVATED WATER TANKS
}

\section{Issam Nasser}

Professor, Department of Structural Engineering, Faculty of Civil

Engineering, Tishreen University, lattakia, Syria.

Email:i.nasser90@yahoo.com .

(Received August 17, 2011 Accepted September 15, 2011)

This research discusses the seismic response of the elevated water tanks which is represented by the base shear forces, displacements and bending moment values, and also studying the dynamic characteristic for this type of especial structures, such as the period and frequencies of vibration, all these are done by dynamic analysis for 3-D mathematical models according to response spectrum, with considering the fluid-structure interactions by representing this fluid with spring-two masses equivalent system: impulsive and convective masses. This analysis has been done by SAP 2000 program. This research involves studying the effect of friction pendulum system (FPS) in dissipating the vibration energy caused by seismic effects on the elevated tanks, and the influence to the response and dynamic specifications values. Also, in this paper the seismic behavior of these elevated tanks which are supplied by tuned mass damper has been evaluated.

A reinforced concrete- elevated water tank has been analyzed and the dynamic analysis results of these seismic isolated tanks showed that the displacements, base shear forces and bending moment generally decreased

KEYWORDS : elevated water tanks; Fluid -structure interaction; passive control; Seismic isolation; Friction pendulum system; tuned mass damper

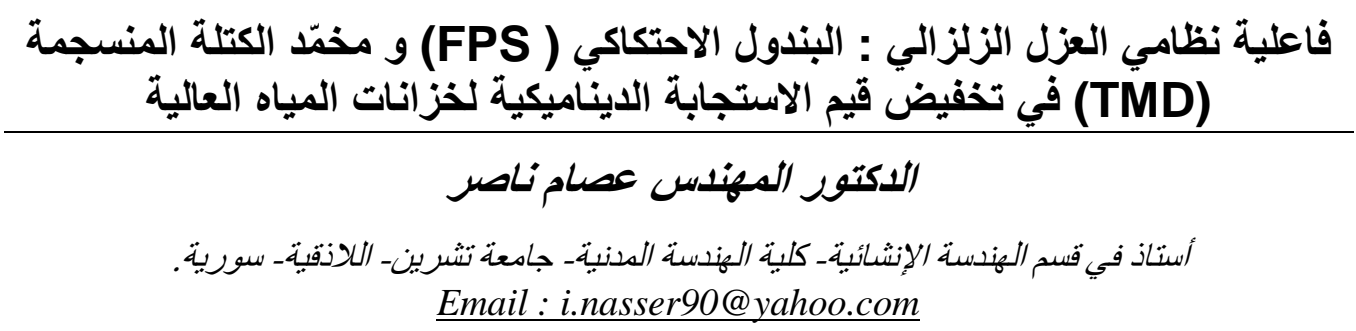

يتتاول البحث مسألة الاستجابة الزلزالية لخزانات الإنات المياه العالية المتمثلة بقيم قوى القص عند مستوى القاعدة ،

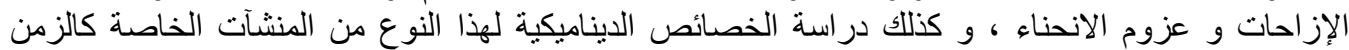

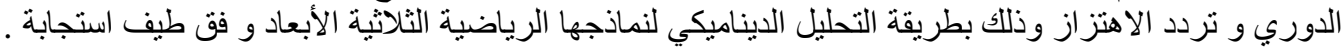

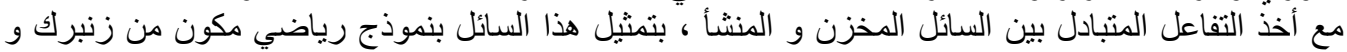

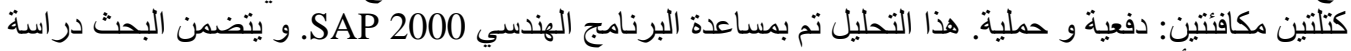
فاعلية إحدى أنظمة العزل عند مستوي القاعدة المتمثل بعازل البندول الاحتكاكي (FPS) في تبديد طاقة الاهتزاز 


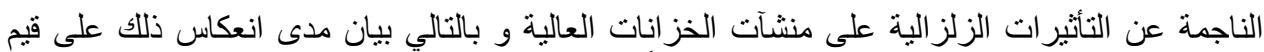

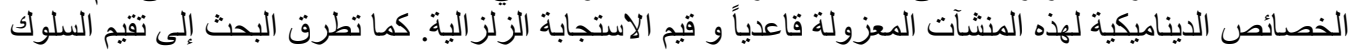

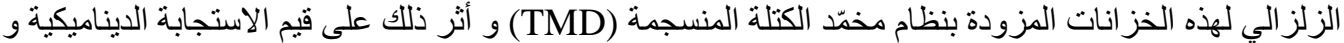

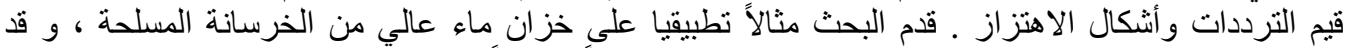

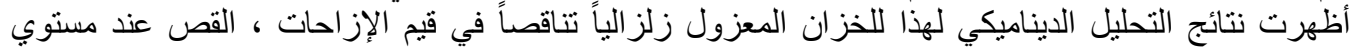

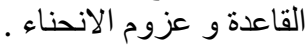

الكلمات المفتاحية : خزانات المباه العالية ، تفاعل سائل ـ منشأ ، التحكم السلبي ، العزل الزلز الي ، عازل البندول الاحتكاكي ، مخمّد الكتلة المنسجمة .

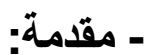

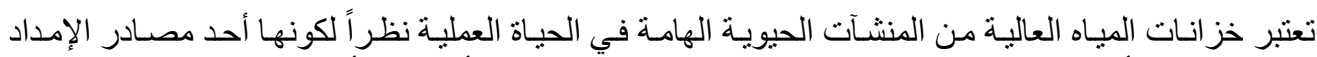

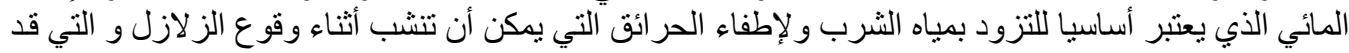

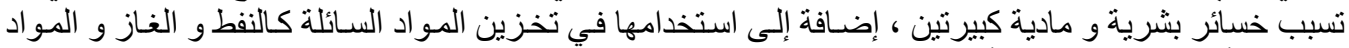

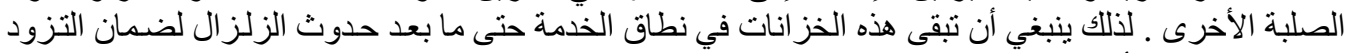

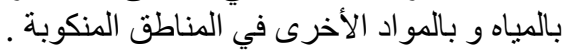

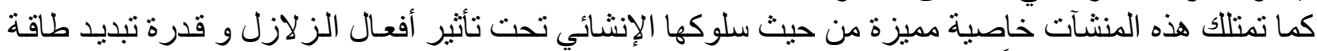

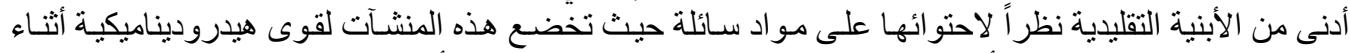

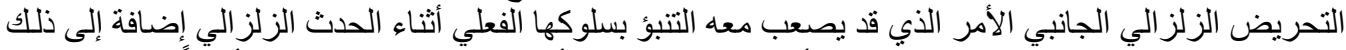

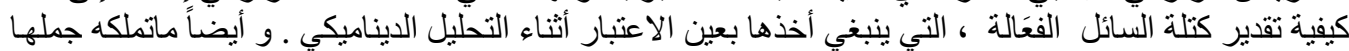

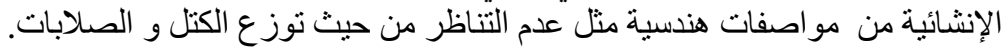

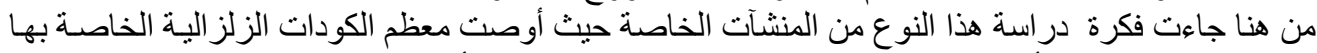

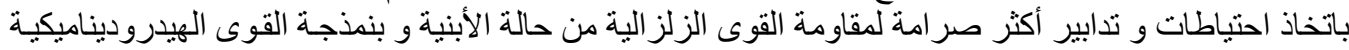

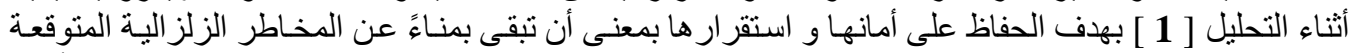

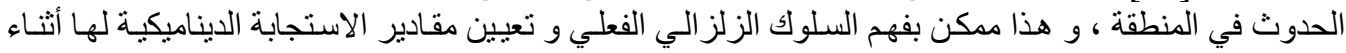

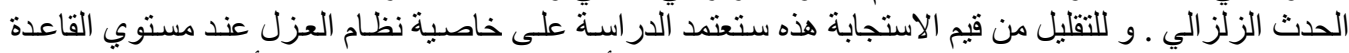

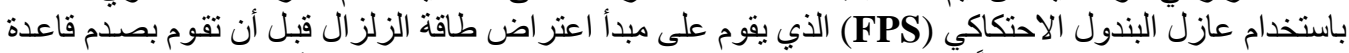

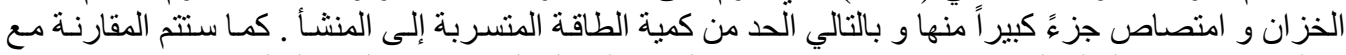

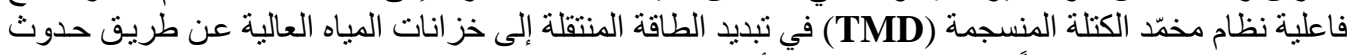

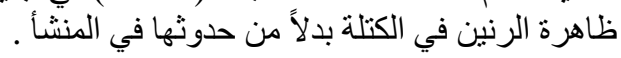

\section{ـ أهمية البحث و أهدافه}

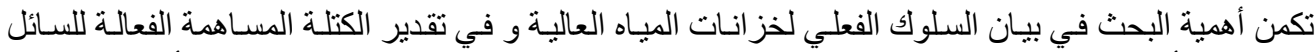

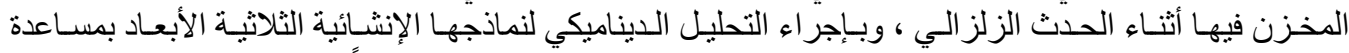

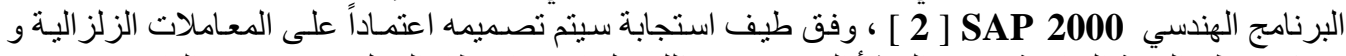

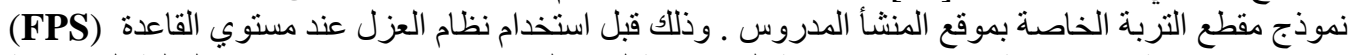

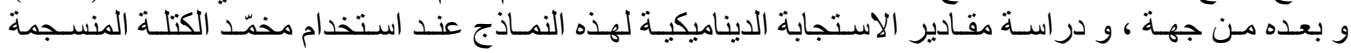

(TMD)

و يهدف البحث إلى بيان مدى فاعلية عازل البندول الاحتكاكي (FPS) في امتصـاص طاقـة الاهنز از المنولدة

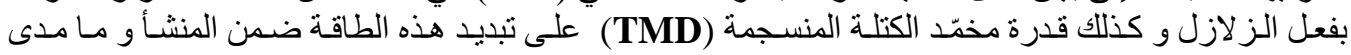

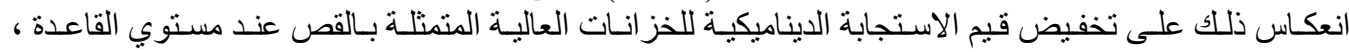

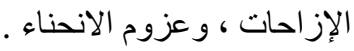




\section{ـ طريقة البحث}

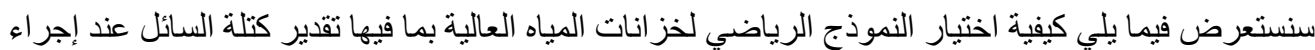

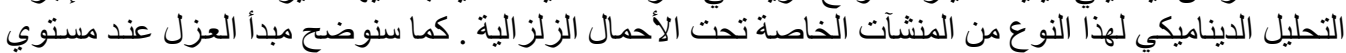

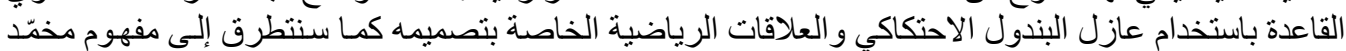

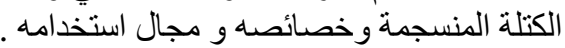

\section{1 - التحليل الديناميكي و النموذج الرياضي للخزانات العالية}

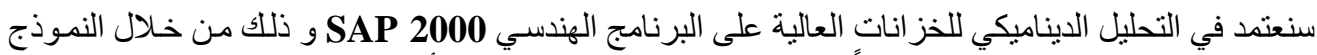

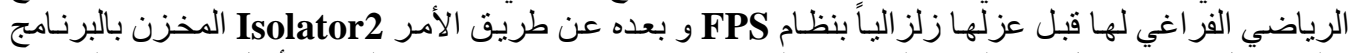

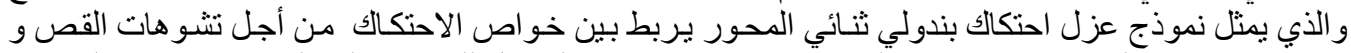

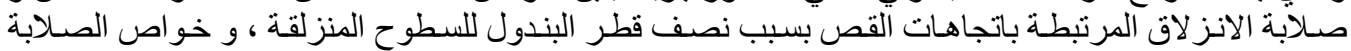

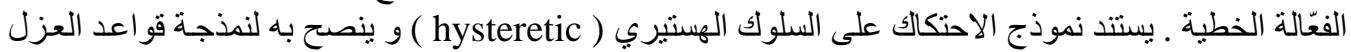

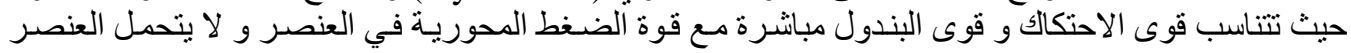

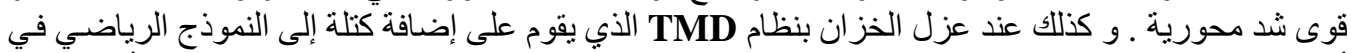

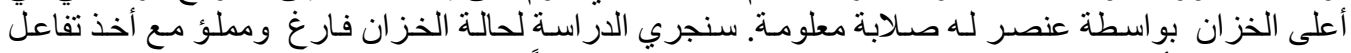

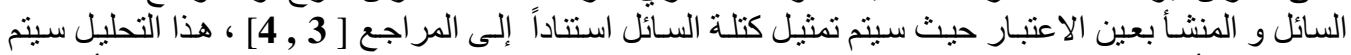

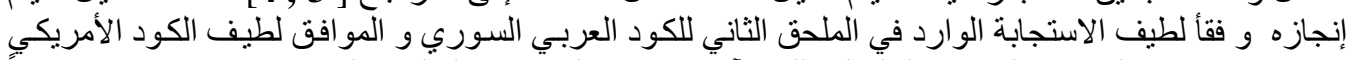

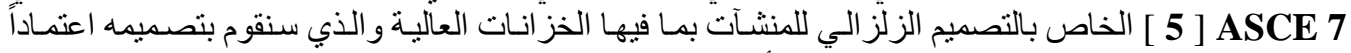

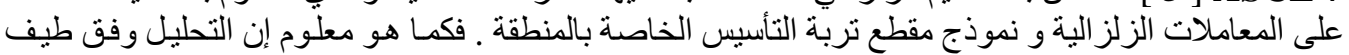

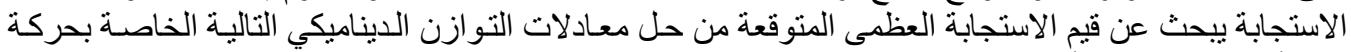

$$
[\mathbf{M}]\{\ddot{\mathbf{u}}\}(\mathbf{t})+[\mathbf{C}]\{\dot{\mathbf{u}}\}(\mathbf{t})+[\mathbf{K}]\{\mathbf{u}\}(\mathbf{t})=[\mathbf{M}]\left[\ddot{\mathbf{y}}_{\mathrm{g}}\right\}(\mathbf{t})
$$
المنشأ الخاضع لحركة أرضية : الانجية

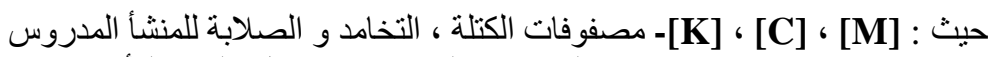

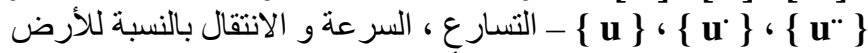

$$
\begin{aligned}
& \text { س }
\end{aligned}
$$

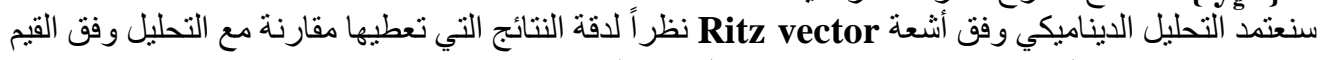

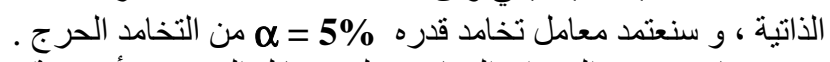

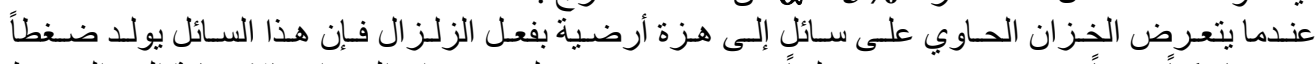

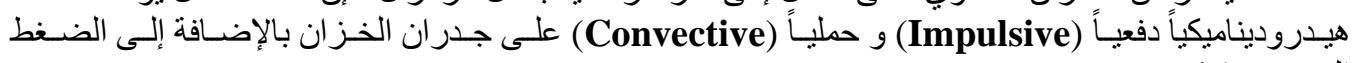

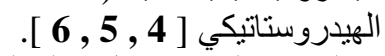

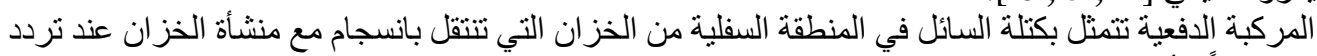

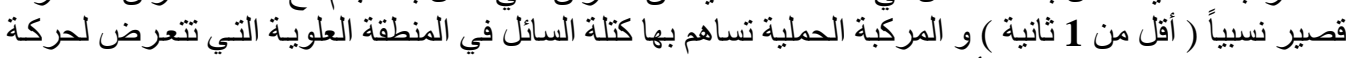

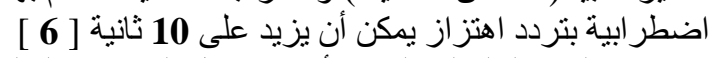

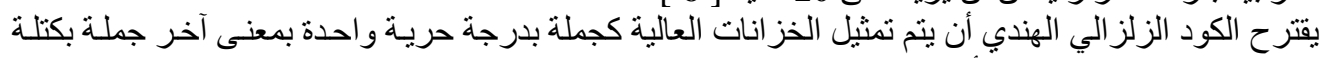

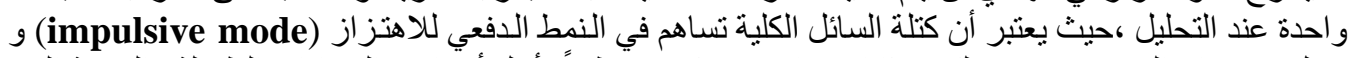

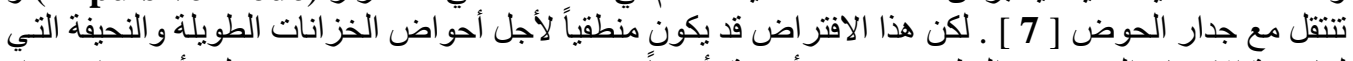

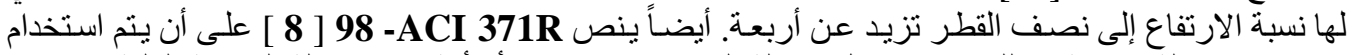
نموذج ذات كتلة مجمعة و ذلك عندما تشكل حمولة الماء التي تتضمن الحمولة الميتة الكلية فوق القاعدة، حمولـة المـاء و

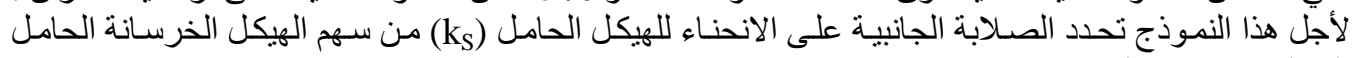

$$
k_{\mathrm{S}}=\frac{3 \mathrm{EI}_{\mathrm{C}}}{\mathbf{L}_{\mathrm{CG}}^{3}}
$$$$
\text { المثل كعمود كابولي [ 8 , } 3 \text { : }
$$ 
حيث : L و - ارتفاع المركز الوسطي للماء المخزن عن القاعدة من E

الزمن الدوري الأساسي للاهنزاز T ينبغي تقييمه في حالة الخز انات العالية و فق الصيغة الآتية [

$$
\mathbf{T}=\mathbf{2} \pi \sqrt{\frac{\mathrm{W}_{\mathrm{L}}}{\mathrm{g} \mathrm{k}_{\mathrm{s}}}}
$$

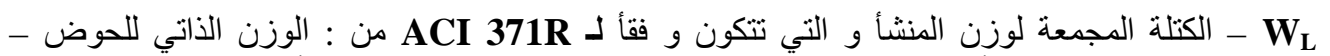
66\% من الوزن الذاتي المنثأ الخرساني الحامل و وزن الماء ، و و - تسارع الجاذبية الأرضية.

\section{2- 20 تفاعل السائل مع المنشأ}

يمكن نمذجة التفاعل المتبادل بين السائل و المنشأ بمقاربات مختلفة [ 4 ] منها : الكتلة المضافة (added mass approach) حيث تتم إضافة الكتلة المكافئة للسائل إلى كتلة المنافئ المنشأ عند السطح

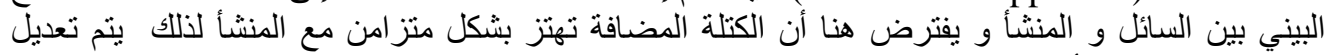

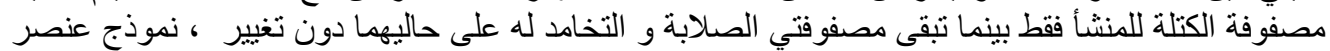

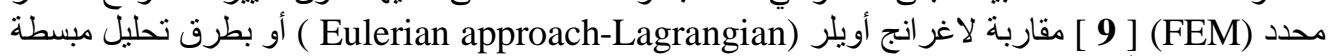

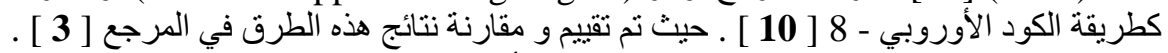

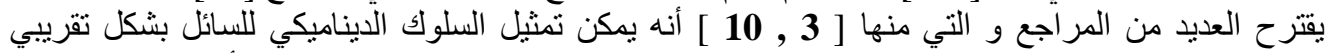

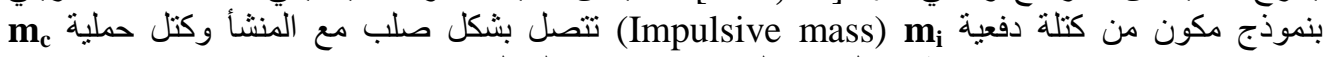
مكافئة تتصل بزنبرك كما هو وارد بالشكل ( 1 (Convective masses)

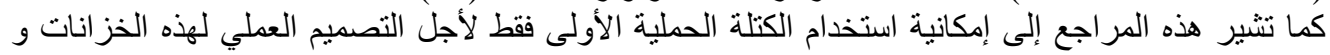

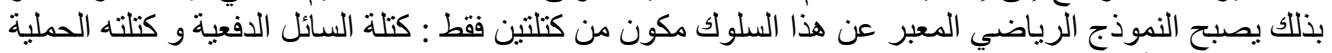

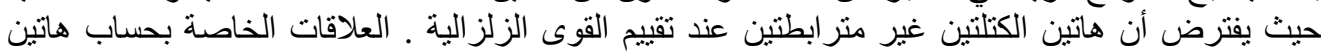

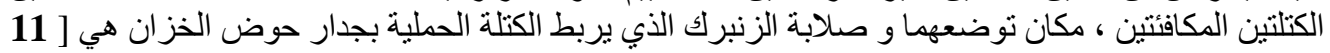

: [,10, 3

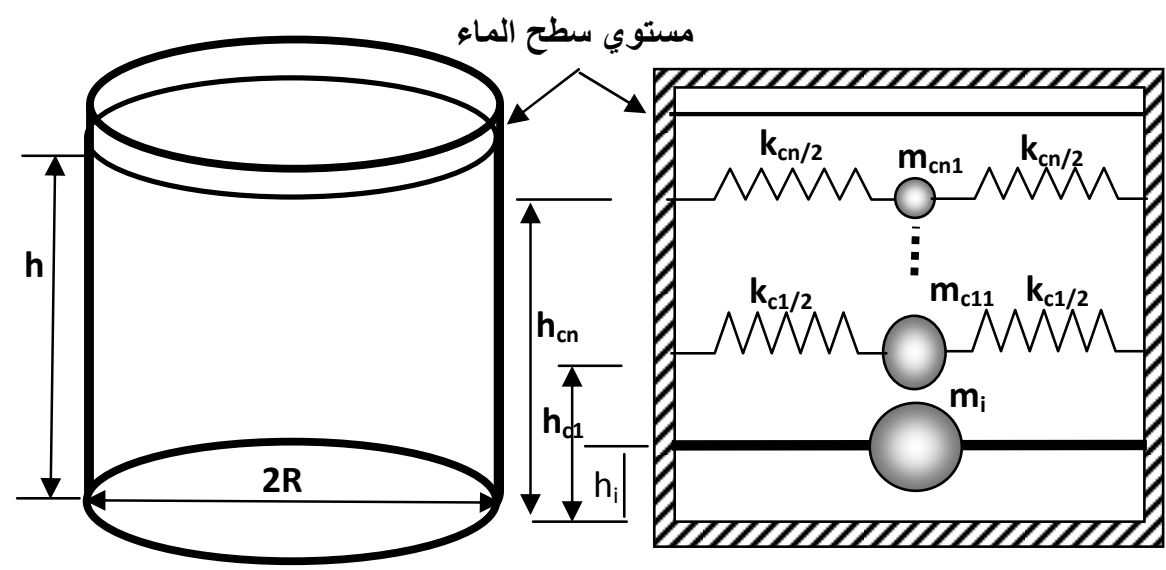

الشكل ( 1 ) - تمثيل خز انات المياه الأسطو انية بجملة كتلة وزنبرك

$$
m_{i}=m_{w} \frac{\tanh (1.74 R / h)}{(1.74 R / h)}
$$

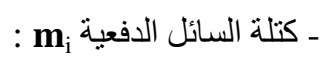




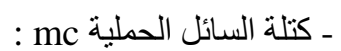

$$
m_{c}=m_{w} \cdot 0.318 \frac{R}{h} \tanh \left(1.84 \frac{h}{R}\right)
$$

- صلابة الزنبرك KC الذي يصل كتلة السائل الحملية بجدار الخزان :

$$
k_{c}=m_{c} \frac{g}{R} 1.84 \tanh \frac{1.84 h}{R}
$$

- منسوب توضع الكتلة الحملية عن أرضية الخزان hc :

$$
h_{c}=\left[1-\frac{\operatorname{Cosh}(1.84 h / R)-1}{(1.84 h / R) \operatorname{Sinh}(1.84 h / R)}\right] h
$$

- منسوب نوضع الكتلة الدفعية عن أرضية الخزان hi :

$$
\mathbf{h}_{\mathrm{i}}=\frac{3}{\mathbf{8}} \mathbf{h}
$$

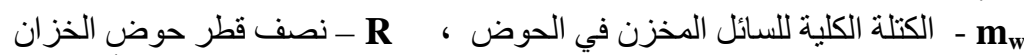
h

\section{3- عازل البندول الاحتكاكي: (FPS)}

يتألف عازل البندول الاحتكاكي من شريحة مغطاة بالتفلون (PTFE) منزلقة على سطح كروي من المعدن المصقول كما في الثكل ( 2 ( ) ) .

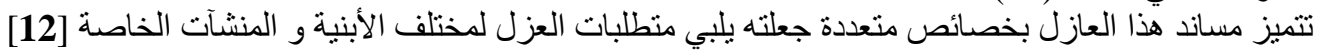

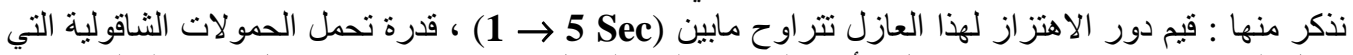

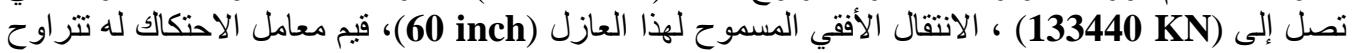

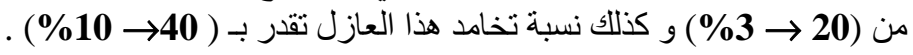

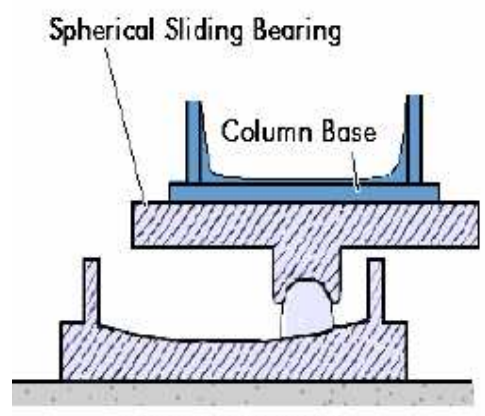

Building Foundation

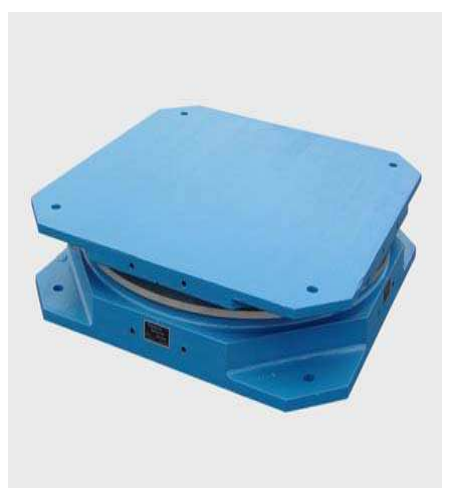

$$
\text { (a)- عازل البندول الاحتكاكيكل ( } 2 \text { ( ) - عازل البندول الاحتكاكي }
$$




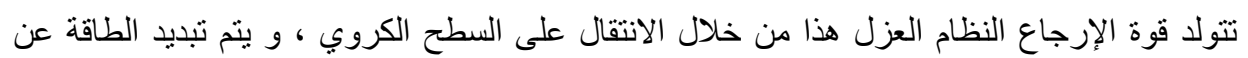

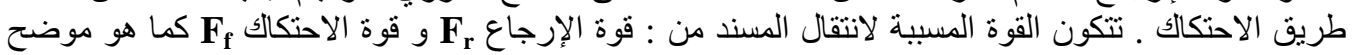
بالثكل

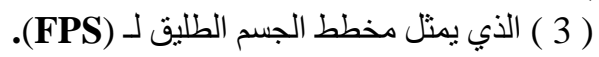

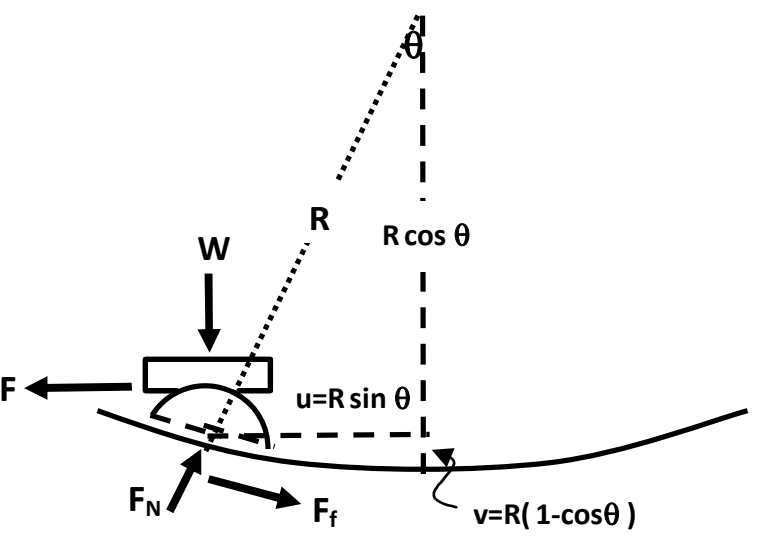

الثكل ( 3) : مخطط الجسم الطليق لعازل البندول الاحتكاكي اعتماداً على مبدأ التوازن نجد أن القوة الأفقية الجانبية F تساوي :

$$
\mathbf{F}=\mathbf{F}_{\mathbf{r}}+\mathbf{F}_{\mathbf{f}}
$$

$$
F=W \tan \theta+\frac{F_{f}}{\cos \theta}
$$

من الشكل ( 3 ) نجد أن هذه القوة تساوي :

حيث : W - W - الوزن المنقول عبر المسند

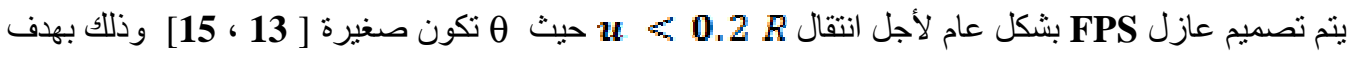
تخفيض خطأحالة التمثيل اللاخطي و لتخفيض القوى المتولدة في العناصر الإنشائية الحاملة للمنشأ في هذه الحالة:

$$
\begin{aligned}
& \sin \theta=\theta-\frac{\theta^{3}}{3 !}+\ldots \approx \theta, \\
& \cos \theta=1-\frac{\theta^{2}}{2 !}+\ldots \approx 1
\end{aligned}
$$

$$
\begin{aligned}
& \tan \theta=\theta=\frac{u}{R \cos \theta} \approx \frac{u}{R} \\
& F_{N}=\frac{W}{\cos \theta} \approx W
\end{aligned}
$$

u = R Sin 


$$
\mathbf{F}_{\mathrm{f}}=\boldsymbol{\mu} \cdot \mathbf{F}_{\mathrm{N}} \operatorname{sgn}(\dot{\mathbf{u}})
$$

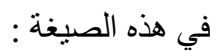

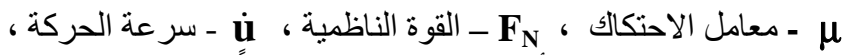

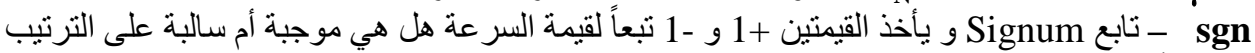

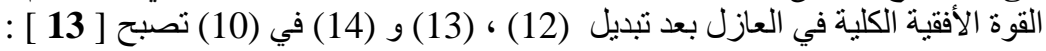

$$
\mathbf{F}=\frac{\mathbf{W}}{\mathbf{R}} \mathbf{u}+\boldsymbol{\mu} \mathbf{W} \cdot \operatorname{sgn}(\dot{\mathbf{u}})
$$

إن قوة الاحتكالك المتولدة على سطح الانزلاق تتعلق بـ : القوة المركزية ، قوة ضغط المسند ، اتجاه و قيمة

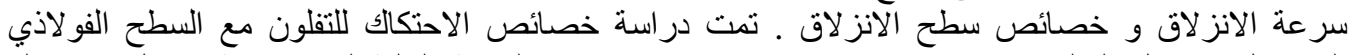
المصقول من قبل العالم Constantinou (1990) حيث اقترح العلافة التالية لتحديد قيمة معامل الاحتكاك

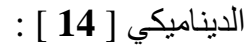

$$
\mu_{S}=f_{\max }-\left(f_{\max }-f_{\min }\right) \exp ^{a|\dot{u}|}
$$

حيث : بs - معامل الاحتكالك بالانز لاق . -

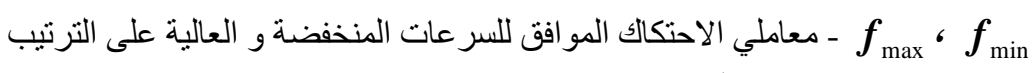
a

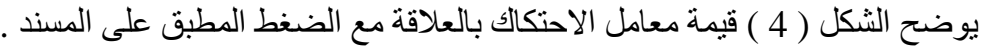

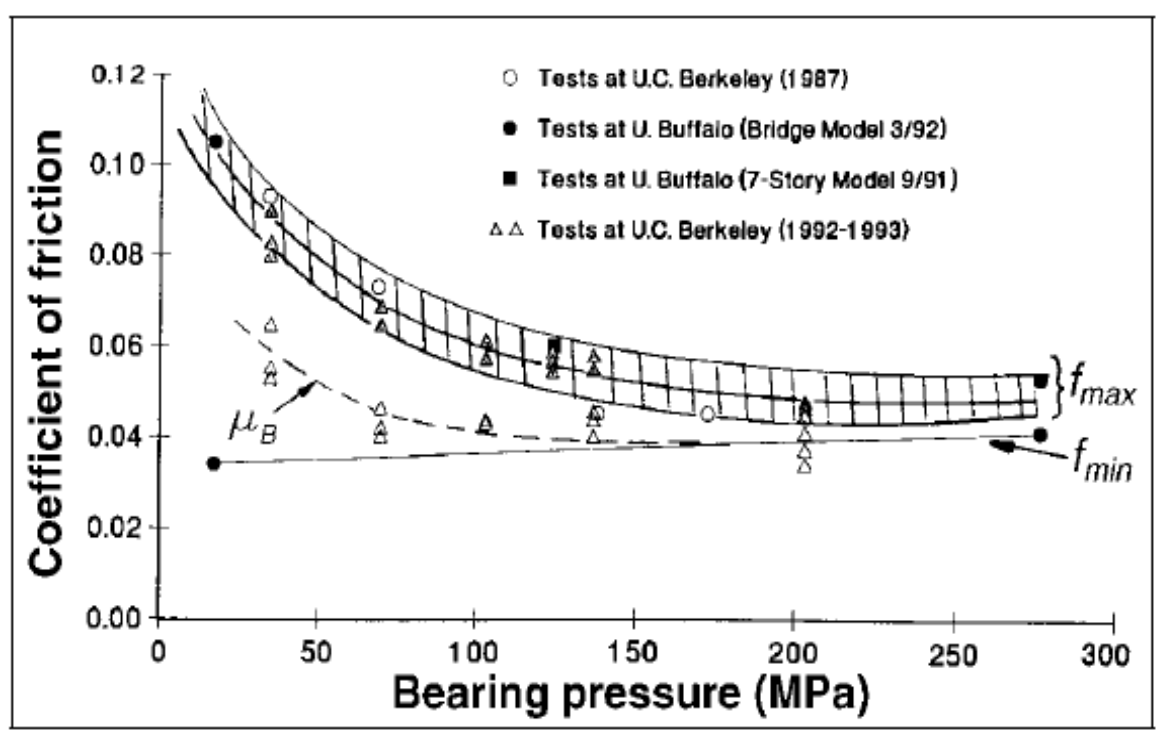

الشكل ( 4 ) - قيم معامل الاحتكالك لمساند FPS بالعلاقة مع الضغط المطبق

الصلابة الفعّالة $\mathbf{K}_{\text {eff }}=\frac{\mathbf{F}}{\mathbf{u}}=\frac{\mathbf{W}}{\mathbf{R}}+\frac{\boldsymbol{\mu} \mathbf{W}}{\mathbf{u}}$ 


$$
\begin{aligned}
& \zeta_{\text {eff }}=\frac{E_{d}}{4 \pi E_{s}}=\frac{4 \mu W u}{4 \pi\left(0.5 K_{\text {eff }} u^{2}\right)}=\frac{2 \mu R}{\pi(\mu R+u)} \\
& E_{S}=0.5 K_{\text {eff }} \mathbf{u}^{2} \text { مساحة الحلقة التخلفية ( الثكل } E_{d}=4 \mu W u \text { u }
\end{aligned}
$$

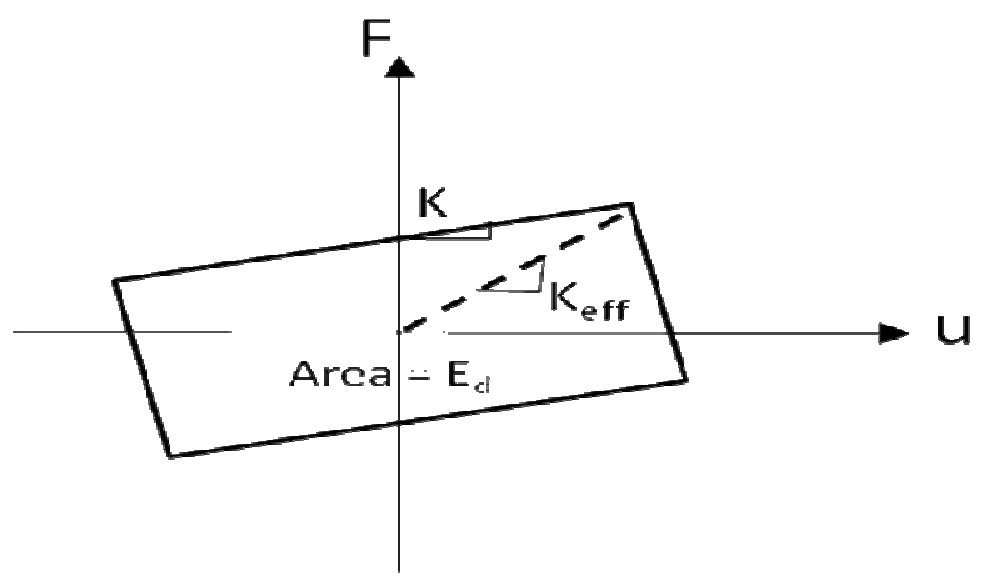

الثكل (5) - الحلقة التخلفية لتمثيل عازل البندول الاحتكاكي

تحسب الصلابة الثشاقولية لنظام العزل وفق الصيغة [ 12 ]:

$$
K_{v}=\frac{W}{g} \cdot\left(\frac{2 \pi}{T_{v}}\right)^{2}
$$

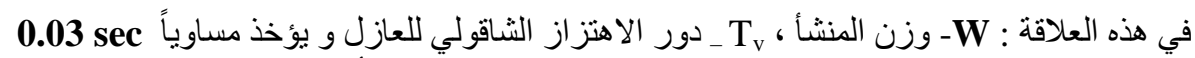

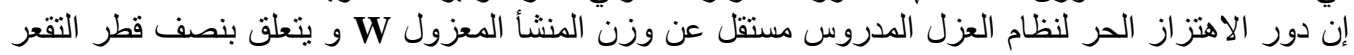

$$
\mathbf{T}=\mathbf{2 \pi} \sqrt{\frac{M}{\mathrm{~K}}}=2 \pi \sqrt{\frac{W}{\mathrm{gK}}}=2 \pi \sqrt{\frac{\mathbf{R}}{\mathrm{g}}}
$$
حيث : خلال الحركة النوسية على السطح المقعر

\section{4- مخمّد الكتلة المنسجمة: (TMD)}

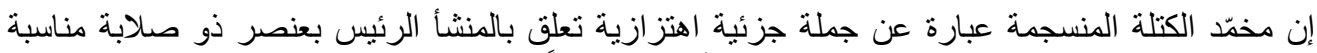

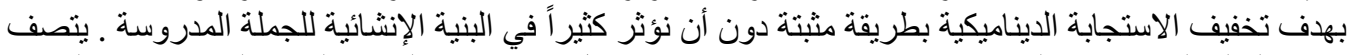

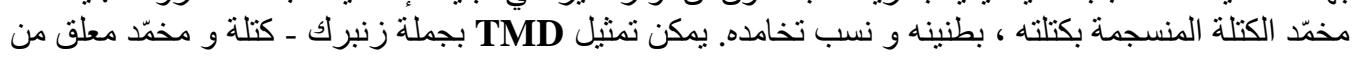


جهة و احدة بالمنشأ الرئيس ، و يفضل أن تتوضع الجملة المضافة هذه عند نقطة محددة بدلاً من أن تكون

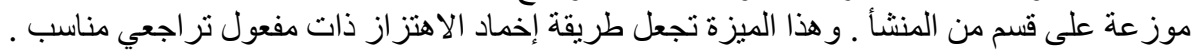

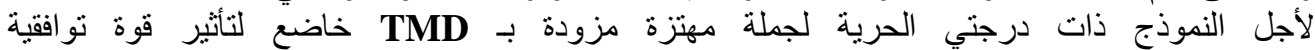

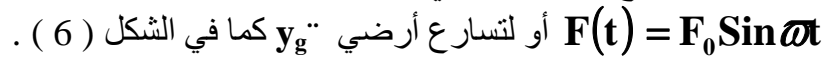

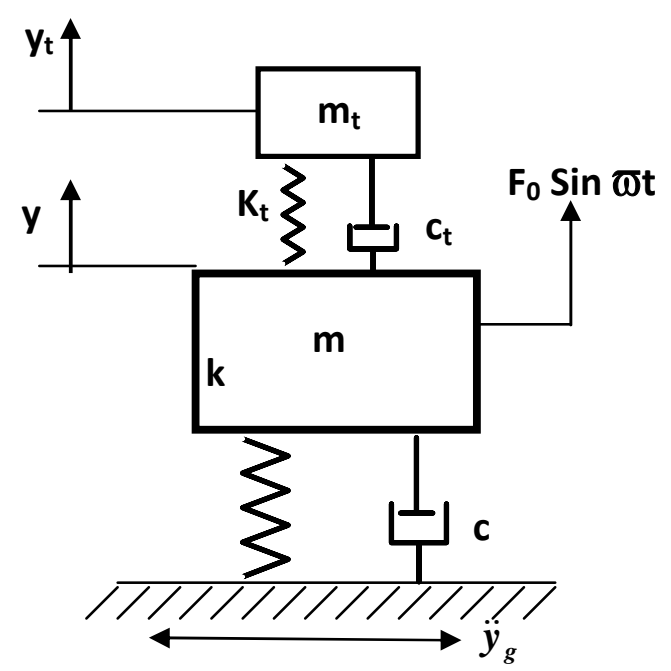

TMD الشكل ( 6 ) - نموذج ذات كتنتين مجمعتين مزود بجملة

$$
\begin{aligned}
& m \ddot{y}+c \dot{y}+k y+c_{t}\left(\dot{y}-\dot{y}_{t}\right)+k_{t}\left(y-y_{t}\right)=F_{0} \operatorname{Sin} \omega t \\
& m_{t} \ddot{\mathbf{y}}_{t}+c_{t}\left(\dot{\mathbf{y}}_{t}-\dot{\mathbf{y}}\right)+k_{t}\left(\mathbf{y}_{t}-\mathbf{y}\right)=0
\end{aligned}
$$

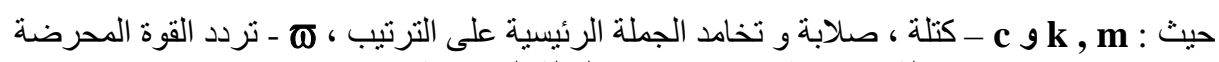

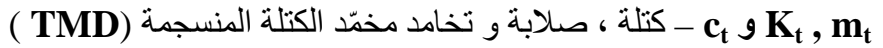

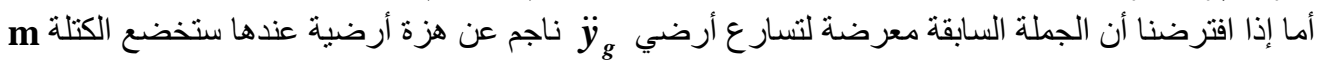

$$
\begin{aligned}
& m \ddot{y}+c\left(\dot{y}-\dot{y}_{g}\right)+k\left(y-y_{g}\right)+c_{t}\left(\dot{y}-\dot{y}_{t}\right)+k_{t}\left(y-y_{t}\right)=0 \\
& m_{t} \ddot{y}_{t}+c_{t}\left(\dot{y}_{t}-\dot{y}\right)+k_{t}\left(y_{t}-\mathbf{y}\right)=0
\end{aligned}
$$

يمكن كتابة العلاقات ( 21 ) و (22 ) بالصيغة المصفوفية:

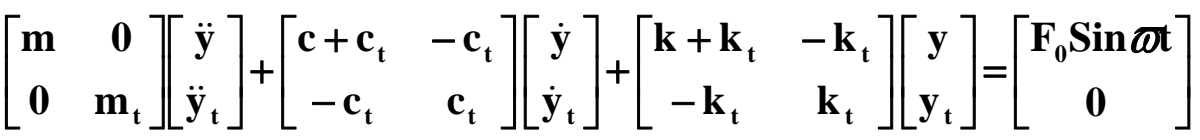

$$
\begin{aligned}
& F(t)=F_{0} e^{i \bar{\omega} t} \\
& \text { يمكن إعطاء التحريض بصيغة أكثر عمومية بحيث يكون مؤلفاً من قسم حقيقي و قسم تخيلي: }
\end{aligned}
$$


$\left[\begin{array}{c}\mathbf{y} \\ \mathbf{y}_{t}\end{array}\right]=\left[\begin{array}{c}\mathbf{Y} \\ \mathbf{Y}_{t}\end{array}\right] \mathbf{e}^{\mathbf{i} \bar{\omega} t}$

و بالتالي المشتق الأول و الثاني بالنسبة للزمن هو:

$$
\left[\begin{array}{c}
\dot{\mathbf{y}} \\
\dot{\mathbf{y}}_{\mathbf{t}}
\end{array}\right]=\mathbf{i} \overline{\boldsymbol{\omega}} \mathbf{e}^{\mathrm{i} \bar{\omega} \mathrm{t}}\left[\begin{array}{c}
\mathbf{Y} \\
\mathbf{Y}_{\mathbf{t}}
\end{array}\right] \cdot\left[\begin{array}{c}
\ddot{\mathbf{y}} \\
\ddot{\mathbf{y}} \mathbf{t}
\end{array}\right]=-\overline{\boldsymbol{\omega}}^{2} \mathbf{e}^{\mathrm{i} \bar{\omega} \mathrm{t}}\left[\begin{array}{c}
\mathbf{Y} \\
\mathbf{Y}_{\mathbf{t}}
\end{array}\right]
$$

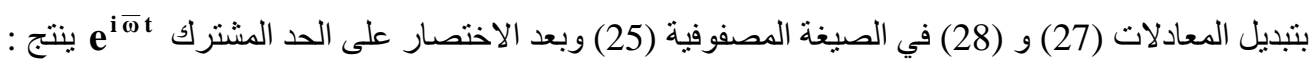

$$
\begin{aligned}
& {\left[\begin{array}{cc}
\left(-\mathbf{m} \bar{\omega}^{2}+k+k_{t}\right)+\left(c+c_{t}\right) \mathbf{i} \bar{\omega} & -k_{t}-c_{t} \mathbf{i} \bar{\omega} \\
-k_{t}-c_{t} i \bar{\omega} & \left(-m_{t} \bar{\omega}^{2}+k_{t}\right)+c_{t} i \bar{\omega}
\end{array}\right]\left[\begin{array}{c}
Y \\
Y_{t}
\end{array}\right]=\left[\begin{array}{c}
F_{0} \\
0
\end{array}\right]} \\
& \text { باستخدام قاعدة كر امر (Cramer's rule) نحصل على السعات : } \\
& \mathbf{Y}=\frac{\left|\begin{array}{cc}
\mathbf{F}_{0} & -\mathbf{k}_{\mathbf{t}}-\mathbf{c}_{\mathbf{t}} \overline{\boldsymbol{\omega}} \mathbf{i} \\
\mathbf{0} & -\mathbf{m}_{\mathbf{t}} \overline{\boldsymbol{\omega}}^{2}+\mathbf{k}_{\mathrm{t}}+\mathbf{c}_{\mathbf{t}} \overline{\boldsymbol{\omega}} \mathbf{i}
\end{array}\right|}{\mathbf{D}}=\frac{-\mathbf{m}_{\mathrm{t}} \overline{\boldsymbol{\omega}}^{2}+\mathbf{k}_{\mathrm{t}}+\mathbf{c}_{\mathrm{t}} \overline{\boldsymbol{\omega}} \mathbf{i}}{\mathbf{D}} \mathbf{F}_{\mathbf{0}} \\
& \mathbf{Y}_{\mathrm{a}}=\frac{\left|\begin{array}{cc}
-\mathbf{m} \overline{\boldsymbol{\omega}}^{2}+\left(\mathbf{c}+\mathbf{c}_{\mathrm{t}}\right) \bar{\omega} \mathbf{i}+\mathbf{k}+\mathbf{k}_{\mathrm{t}} & \mathbf{F}_{0} \\
-\mathbf{c}_{\mathrm{t}} \overline{\boldsymbol{\omega}} \mathbf{i}-\mathbf{k}_{\mathrm{t}} & \mathbf{0}
\end{array}\right|}{\mathbf{D}}=\frac{\mathbf{k}_{\mathrm{t}}+\mathbf{c}_{\mathrm{t}} \overline{\boldsymbol{\omega}} \mathbf{i}}{\mathbf{D}} \mathbf{F}_{\mathbf{0}} \\
& \mathbf{D}=\left(-\mathbf{m} \overline{\boldsymbol{\omega}}^{2}+\left(\mathbf{c}+\mathbf{c}_{\mathrm{t}}\right) \overline{\boldsymbol{\omega}} \mathbf{i}+\mathbf{k}+\mathbf{k}_{\mathrm{t}}\right)\left(-\mathbf{m}_{\mathbf{t}} \overline{\boldsymbol{\omega}}^{2}+\mathbf{c}_{\mathbf{t}} \overline{\boldsymbol{\omega}} \mathbf{i}+\mathbf{k}_{\mathrm{t}}\right)-\left(\mathbf{c}_{\mathrm{t}} \overline{\boldsymbol{\omega}} \mathbf{i}+\mathbf{k}_{\mathrm{t}}\right)^{2}
\end{aligned}
$$

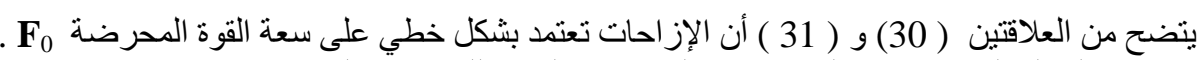

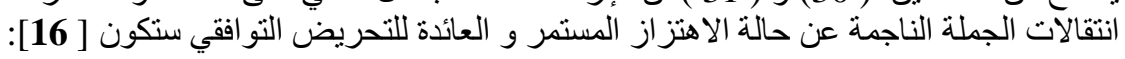

$$
\begin{aligned}
& |\mathbf{Y}(\mathbf{i} \overline{\boldsymbol{\omega}})|=\left|\frac{-\mathbf{m}_{\mathbf{t}} \overline{\boldsymbol{\omega}}^{2}+\mathbf{k}_{\mathbf{t}}+\mathbf{c}_{\mathbf{t}} \overline{\boldsymbol{\omega}} \mathbf{i}}{\mathbf{D}(\mathbf{i} \overline{\boldsymbol{\omega}})} \mathbf{F}_{\mathbf{0}}\right| \\
& \left|\mathbf{Y}_{\mathbf{t}}(\mathbf{i} \overline{\boldsymbol{\omega}})\right|=\left|\frac{\mathbf{k}_{\mathbf{t}}+\mathbf{c}_{\mathbf{t}} \overline{\boldsymbol{\omega}} \mathbf{i}}{\mathbf{D}(\mathbf{i} \overline{\boldsymbol{\omega}})} \mathbf{F}_{\mathbf{0}}\right|
\end{aligned}
$$

بهذف كتابة العلاقة ( 32 ) بالصيغة الأكثر ملاءمة نعرف ما يلي :

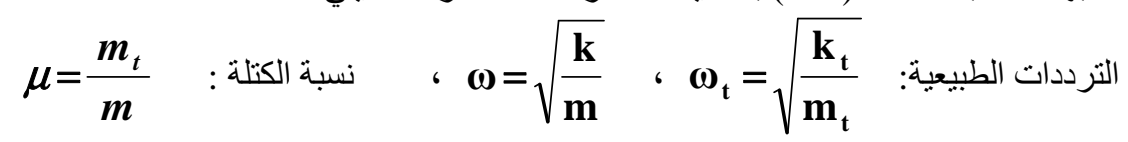

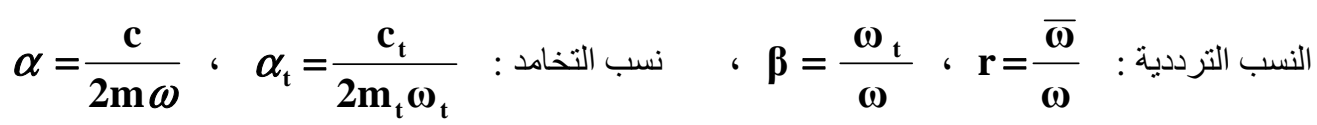

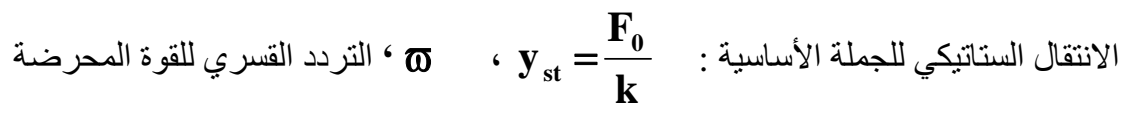




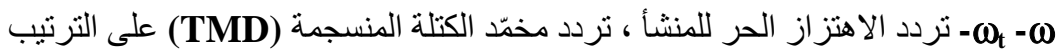

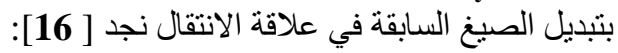

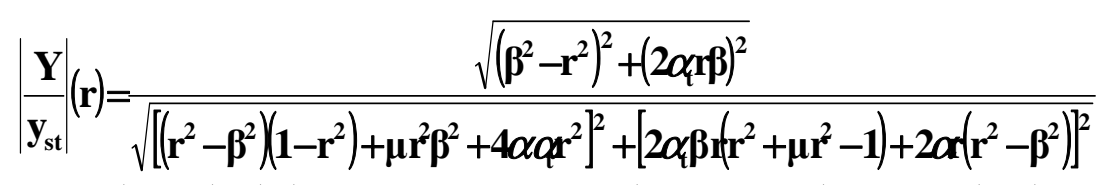

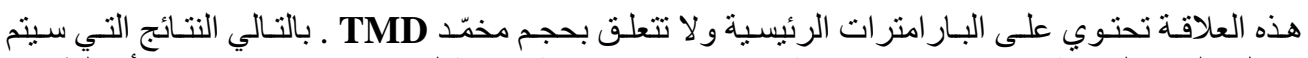

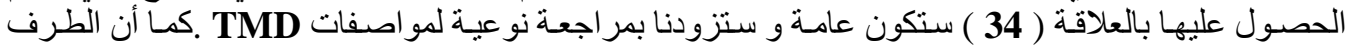

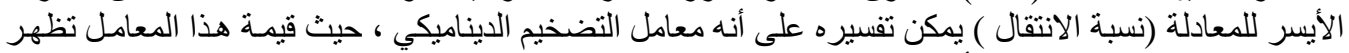

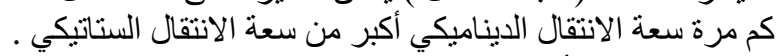

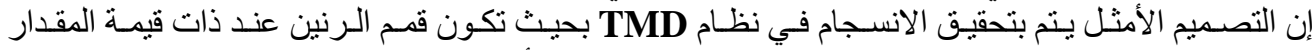

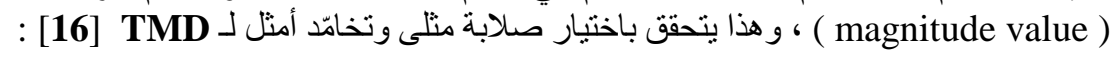

$$
\begin{gathered}
\beta_{\text {opt }}=\frac{1}{1+\mu} \\
\alpha_{\text {topt }}=\sqrt{\frac{3 \mu}{8(1+\mu)}}
\end{gathered}
$$

تشير العلاقة (35) إلى أن رنين يحدث دائما عند نردد منخفض أقل من التردد الطبيعي للجملة الأساسية

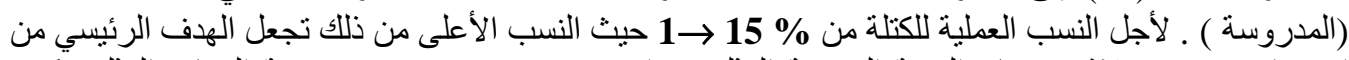
استخدام TMD يتلاشى ، فإن النسبة الترددية المنلى نتراوح بين 0.87 - 0.99 و نسبة التخامد المنلى تكون

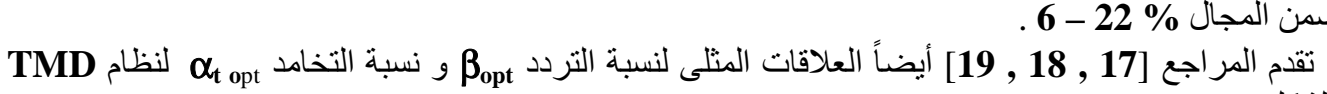

$$
\begin{aligned}
& \beta_{\text {opt }}=\frac{1}{1+\mu}\left(1-\alpha \sqrt{\frac{\mu}{1+\mu}}\right) \\
& \alpha_{\mathrm{topt}}=\frac{\alpha}{1+\mu}+\sqrt{\frac{\mu}{1+\mu}}
\end{aligned}
$$

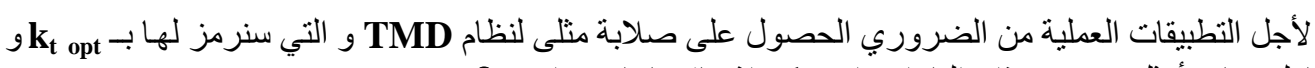

معامل تخامد أمثل ct opt هذان البار امنر ان يمكن اشتقاقهما باستخدام

$$
\begin{gathered}
k_{\text {topt }}=m_{t} \omega^{2} \beta_{t o p t}{ }^{2}=\frac{m_{t} \omega^{2}}{(1+\mu)^{2}}\left(1-\frac{\alpha}{1+\mu} \sqrt{\frac{\mu}{1+\mu}}\right)^{2} \\
c_{t o p t}=2 m_{t} \omega \beta_{o p t} \alpha_{t o p t}=\frac{2 m_{t} \omega}{1+\mu}\left(1-\alpha \sqrt{\frac{\mu}{1+\mu}}\right)\left(\frac{\alpha}{1+\mu}-\sqrt{\frac{\mu}{1+\mu}}\right)
\end{gathered}
$$

يقترح المرجع [ 20 ] أن تكون النسبة بين صلابة الر ابط المرن (k إب) الحامل للكتلة المخمّدة و بين الكتلة المخمّدة

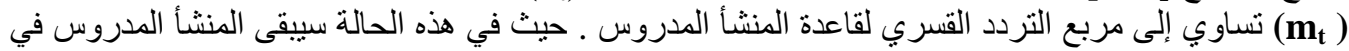

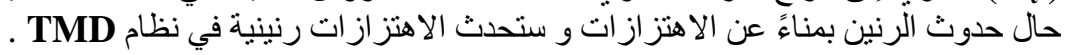




\section{النتائج والمناقشة}

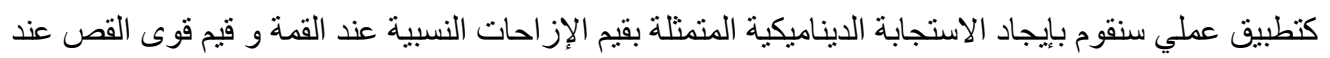

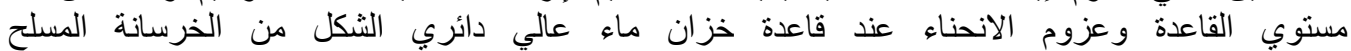
(الثكل 7) سعته

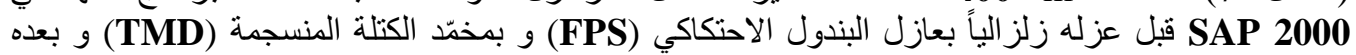

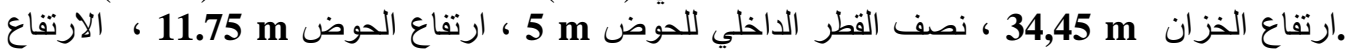

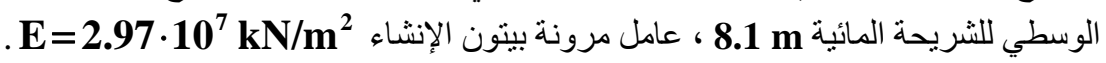

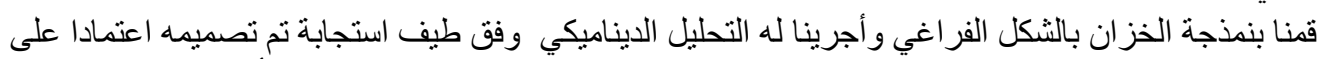

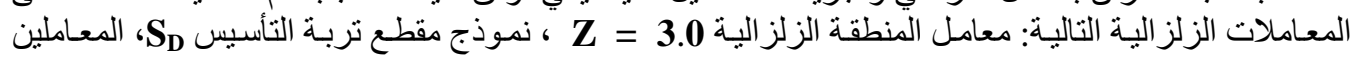

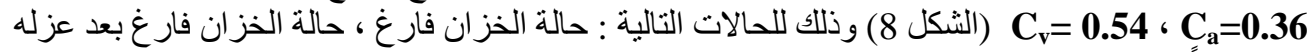

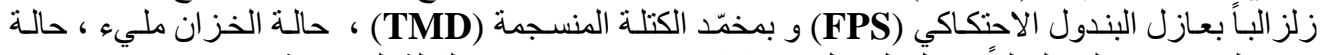

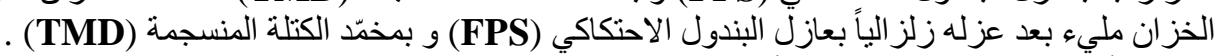

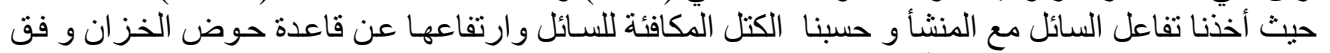
العلاقات ( 4 - 8) و كانت القيم كالآتي: كتلة الماء في حوض الخزان التان:

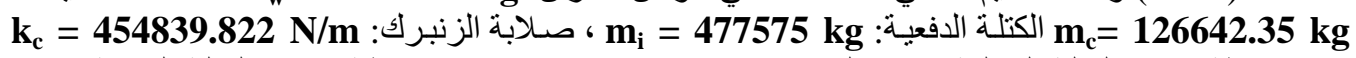
ارتفاع نقطة تركيز الكتلة الحملية ضمن الحوض: ho الحوض: ho4

\section{- FPS تصميم نظام العزل}

ـ الصلابة الأولية للعازل :

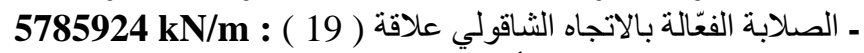

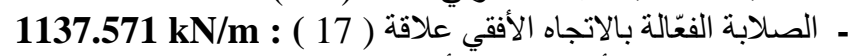

ـ معامل الاحتكاك الأصغري و الأعظمي : 0.06 ـ 0.09 ـ

\section{- تصميم نظام TMD -}

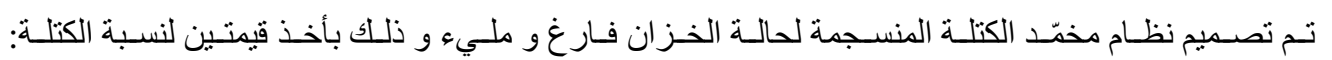

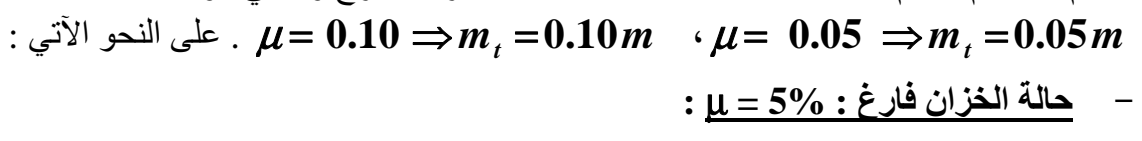

$$
\begin{aligned}
& \text { ، } m=\frac{W}{g}=\frac{6852}{9.81}=698.47 \mathrm{kN} . \mathrm{Sec}^{2} / \mathrm{m} \text { : كتلة الخزان } \\
& m_{t}=0.05 \cdot 698.47=34.92 \approx 35 \mathrm{kN} \cdot \mathrm{Sec}^{2} / \mathrm{m} \text { كتلة المخمّد : } \\
& \text { - } \\
& \alpha_{\text {topt }}=\sqrt{\frac{3 \cdot 0.05}{8(1+0.05)}}=0.134: \text { : نسبة التخامد المثلى - }
\end{aligned}
$$

$: \ddot{a}$

الكرك لملابة المثل

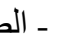

$$
k_{\text {topt }}=m_{t} \omega^{2} \beta_{\text {topt }}{ }^{2}=35 \cdot(11.619)^{2}(0.952)^{2}=4282.32 \mathrm{kN} / \mathrm{m}
$$




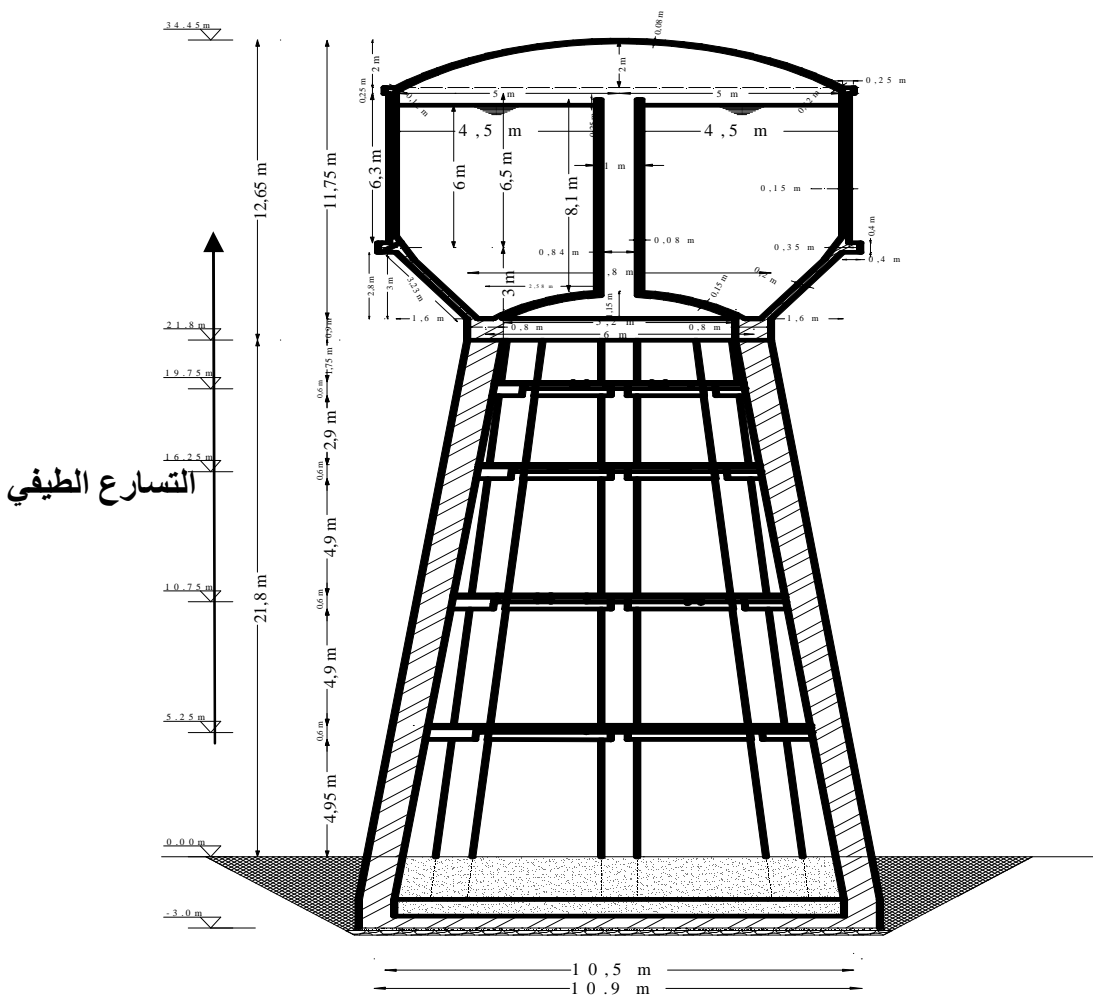

الثكل ( 7 ) : مقطع رأسي في هيكل الخزان العالي

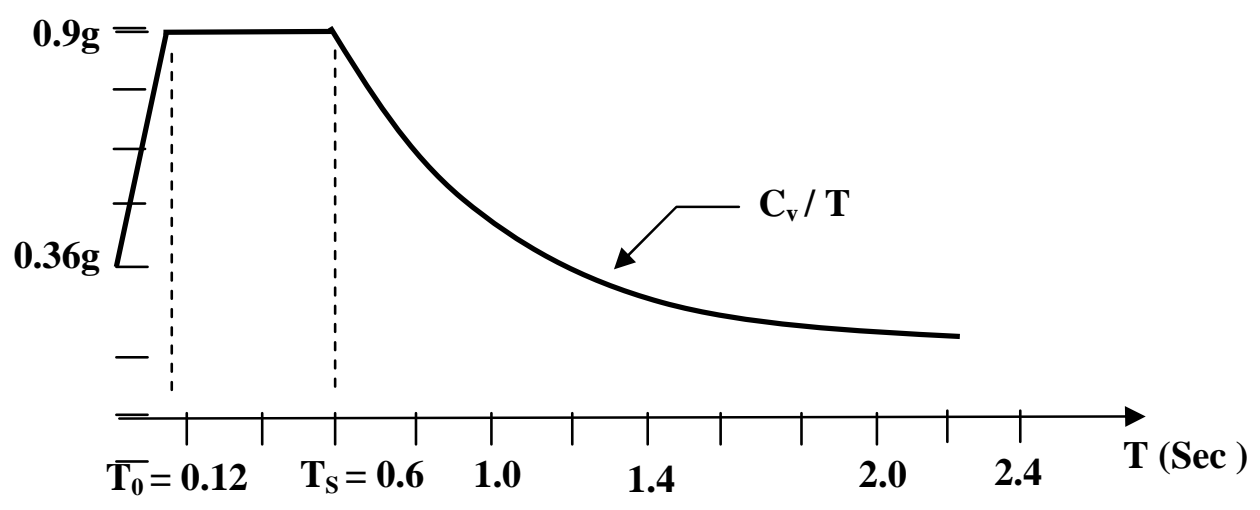

الثكل ( 8 ) ) : طيف الاستجابة التصميمي

\section{- حالة الخزان فارغ :} ، m = 698.47 kN. Sec²/m : $m_{t}=0.10$ - $698.47=69.85$ kN. Sec 2 /m : $\beta_{\text {opt }}=\frac{1}{1+0.10}=0.91 \quad$ : نسبة التردد المثلى : 


$$
\alpha_{\text {topt }}=\sqrt{\frac{3 \cdot 0.10}{8(1+0.10)}}=0.184: \text { : نسبة التخامد المثلى - }
$$

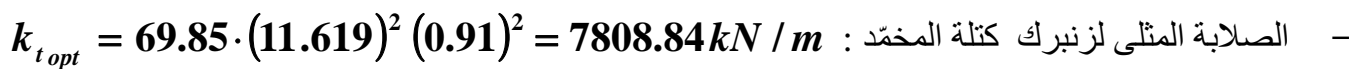

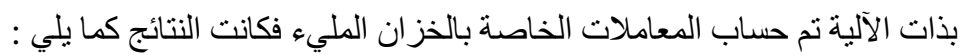
- بالة الخزان ملىء :

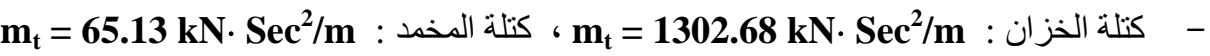
$\alpha_{\text {opt }}=0.134$ - ن ن $k_{\text {topt }}=65.13 \cdot(2.573)^{2}(0.952)^{2}=390.78 k N / m$ : - -

m $m_{t}=130.27$ kN $\cdot$ Sec$^{2} / m$ : كتلة الخزان : $\alpha_{\text {opt }}=0.184$ : نسبة التردد المنلى :

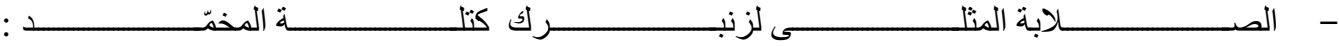

$$
k_{\text {topt }}=130.27 \cdot(2.573)^{2}(0.910)^{2}=714.16 \mathrm{kN} / \mathrm{m}
$$

قمنا بإضافة نظام TMD إلى قمة الخزان و أعدنا التحليل الديناميكي للحالتين الدذكورتين .

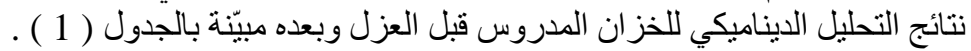

\begin{tabular}{|c|c|c|c|c|c|c|c|}
\hline \multicolumn{2}{|c|}{$\begin{array}{l}\text { عزوم الانحناء } \\
\text { (k N.m ) } \\
\end{array}$} & \multicolumn{2}{|c|}{ القص عند مستوي القاعدة } & \multicolumn{2}{|c|}{$\begin{array}{c}\text { الإزاحات } \\
\text { ( m ) } \\
\end{array}$} & \multirow{2}{*}{$\begin{array}{c}\text { الاهتزاز } \\
\text { (Sec) } \\
\end{array}$} & \\
\hline$(\mathbf{y})$ & $(\mathbf{x})$ & $(\mathbf{y})$ & $(\mathbf{x})$ & $(\mathbf{y})$ & $(\mathbf{x})$ & & \\
\hline 83884.33 & 83929.17 & 3695.24 & 3688.88 & 0.066 & 0.066 & 0.541 & الخزان فالقارعدة \\
\hline 32905.76 & 32897.23 & 2284.10 & 2283.73 & 0.227 & 0.227 & 1.617 & معزول بنظام فارغ و \\
\hline 79069.19 & 79571.54 & 3431.99 & 3369.21 & 0.061 & 0.061 & 2.183 & 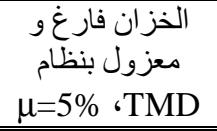 \\
\hline 92723.49 & 86835.08 & 3492.13 & 3505.05 & 0.069 & 0.069 & 2.579 & 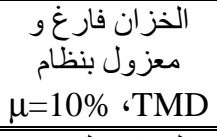 \\
\hline 387857.25 & 387884.66 & 13818.08 & 13815.30 & 0.304 & 0.304 & 2.441 & مثبت بالقاعدة وليء \\
\hline 174612.82 & 177335.46 & 6535.78 & 6414.45 & 0.719 & 0.706 & 5.416 & معزول بنزام مليء و \\
\hline 321583.02 & 381977.05 & 13640.58 & 11490.61 & 0.30 & 0.252 & 8.217 & 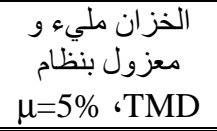 \\
\hline 317536.88 & 378315.18 & 13523.28 & 11355.20 & 0.296 & 0.248 & 8.753 & 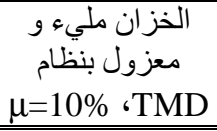 \\
\hline
\end{tabular}
الجدول ( 1) قيم الاستجابة الناتجة عن التحليل الديناميكي للخزان المدروس النيك 
أنماط الاهتز از الأربعة الأولى لحلة الخزان مليء قبل عزله بنظام FPS و بنظام TMD و بعده معطاة

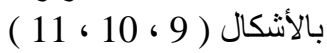

\section{SAP2000- - ist}

Fle Edit Yiew Define Binge Dlaw Select Assign Analyze Display Design Options Help

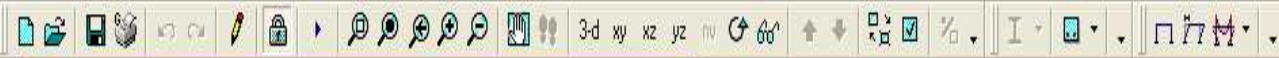

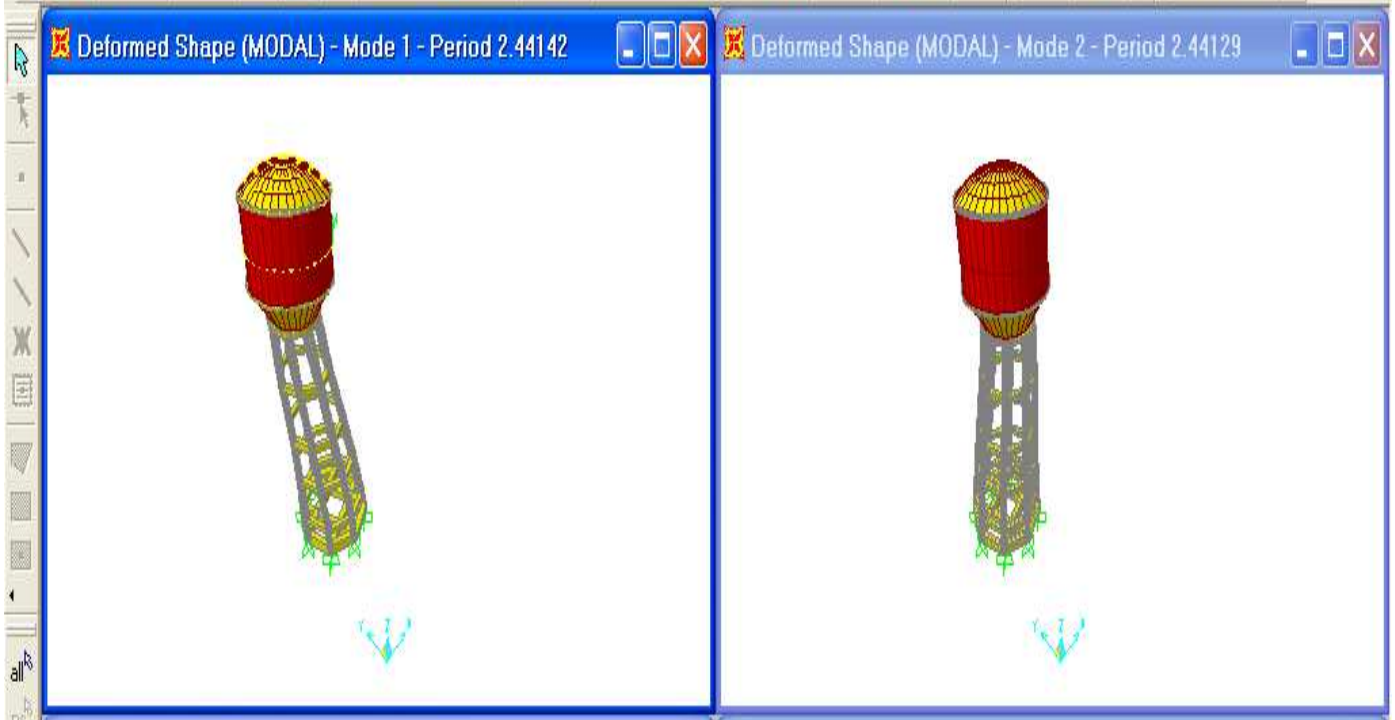

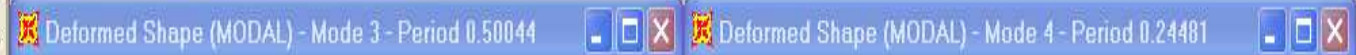
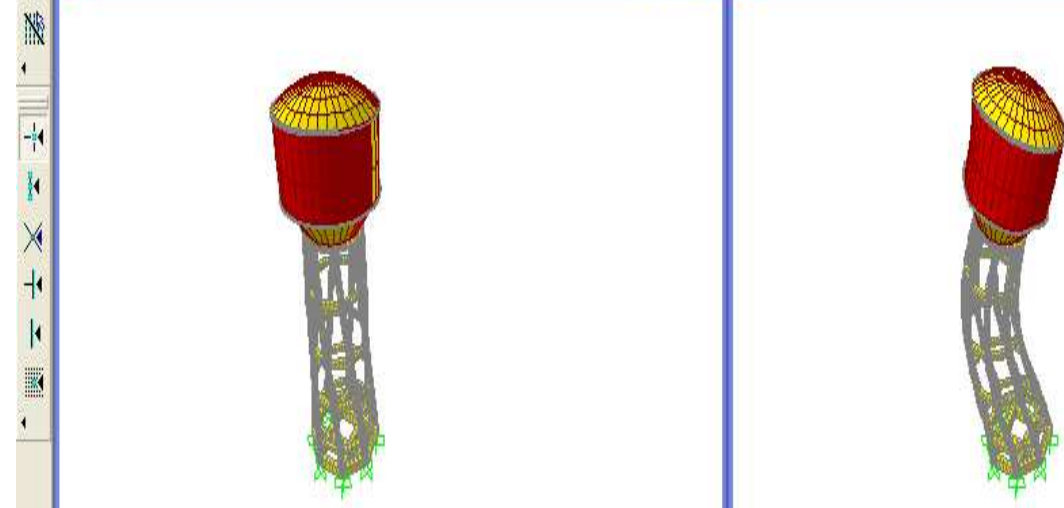

Right Click on any jont for displacerment values

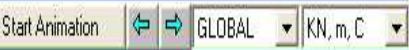

$12033 \div$ ? 3 EN :

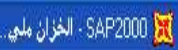

$$
\text { الشكل ( } 9 \text { ) - الأنماط الأربعة الأولى لحالة الخزان مليء ومثبت بالأرض }
$$




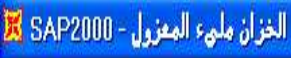

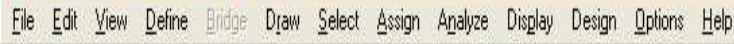

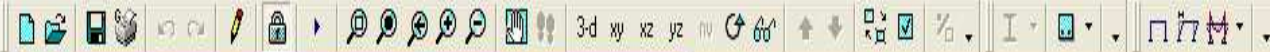

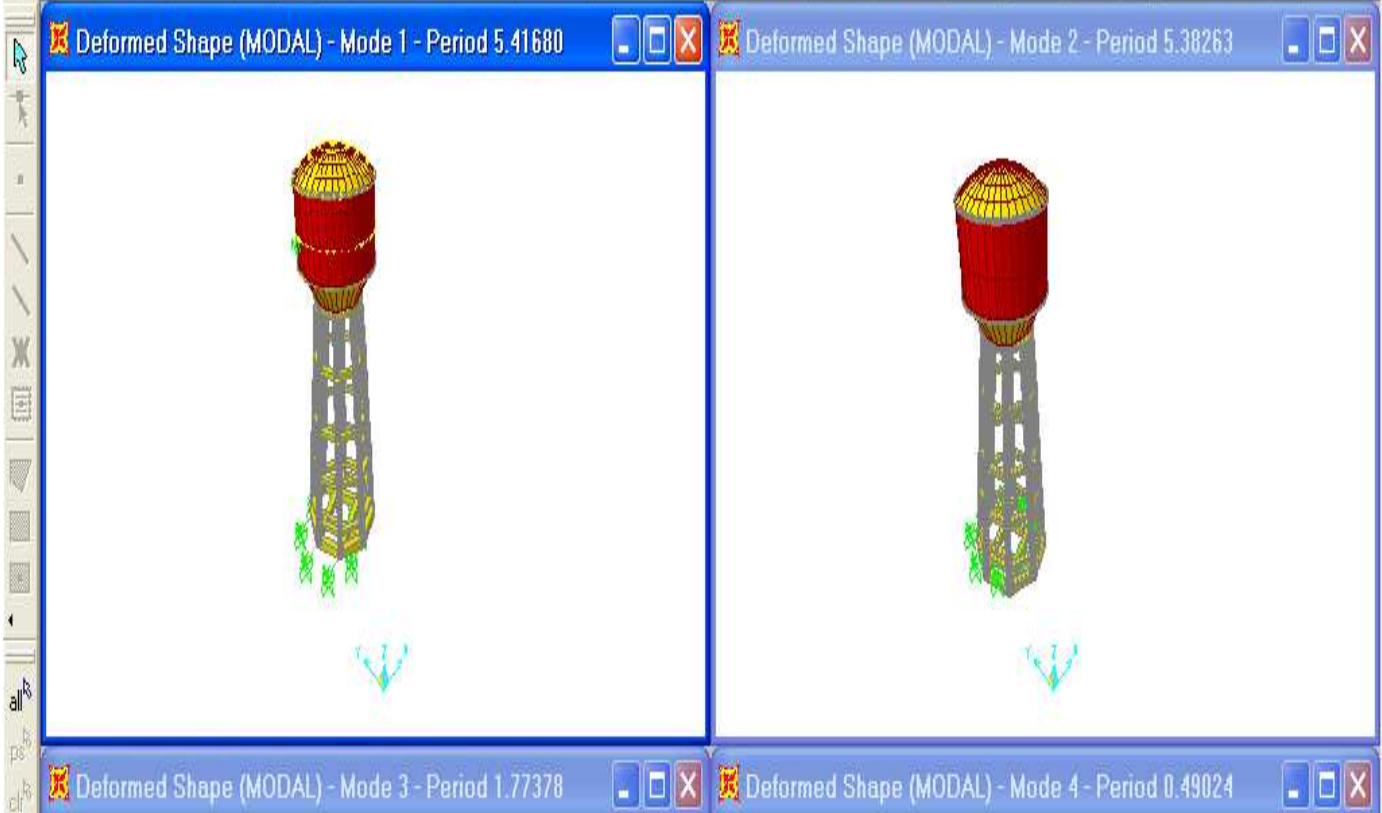

(1)
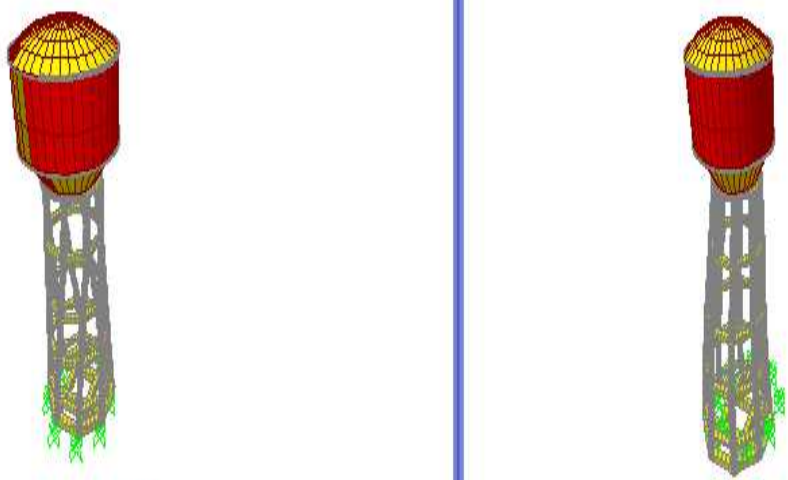

Right Click on any joint for displacement values

Statidinimation $\Rightarrow \Rightarrow$ GLOBAL $\rightarrow$ Kgl, mm, C V

+1210 (3) 5 . $\mathrm{EN}:$

FPS الثكل ( 10 ) - الأنماط الأربعة الأولى لحالة الخزان مليء و معزول بنظام 


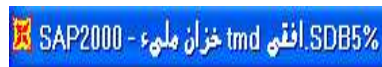

[. [.]

Fle Edot Yiew Define Bidge Diaw Select Assign Anayze Display Design Opplons Help

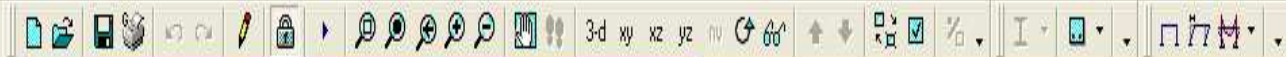

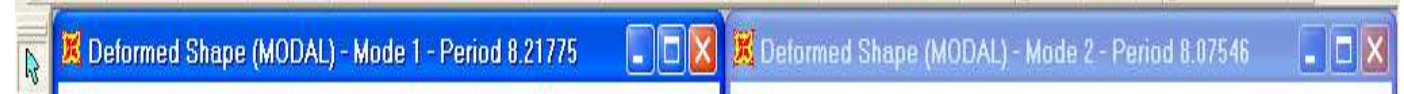

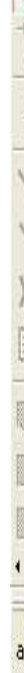

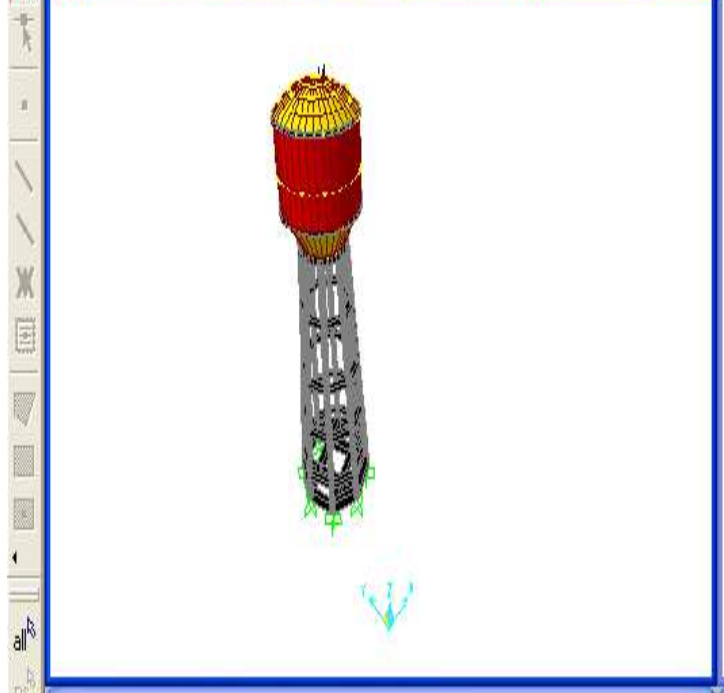

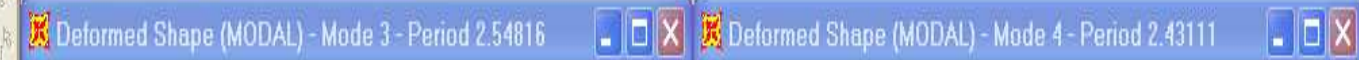

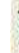

14

Right Click on any jon for displacement values

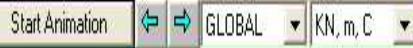

1217 (7) ? ? EN
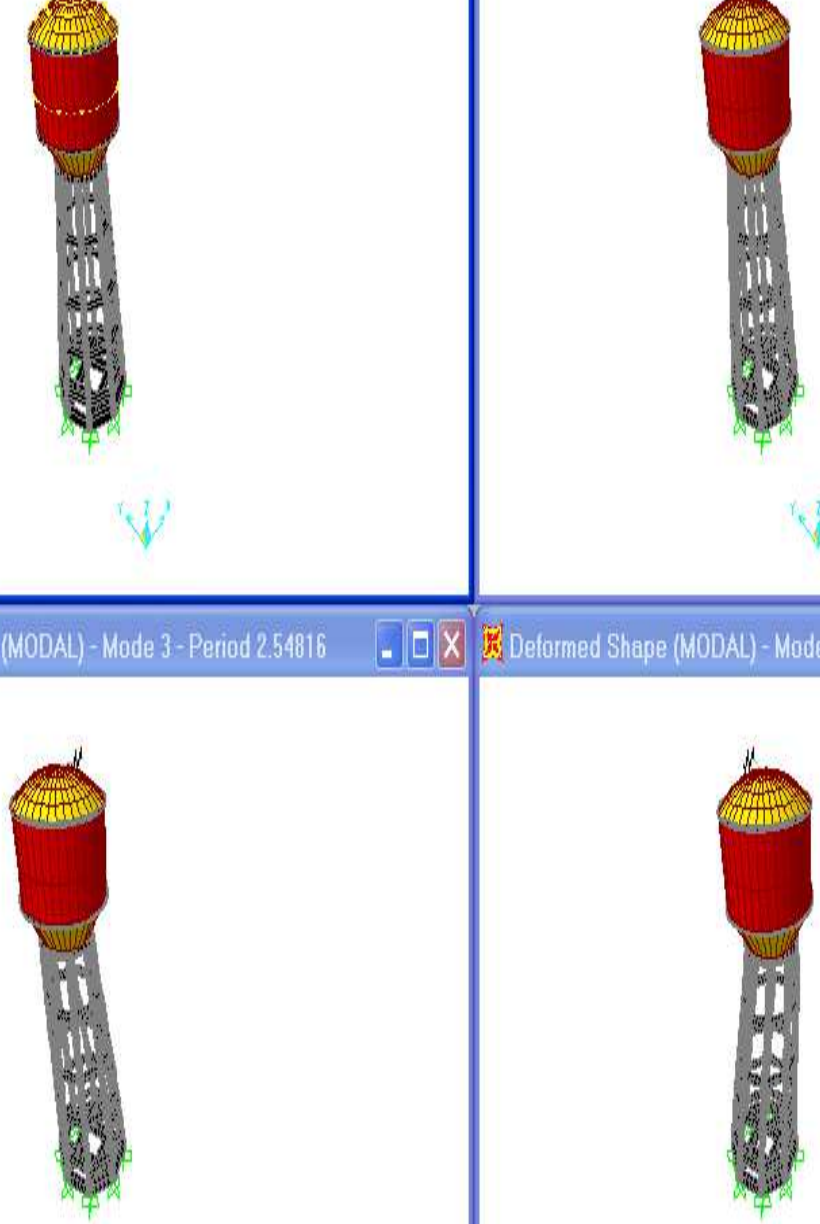


\section{الاستتتاجات و التوصيات}

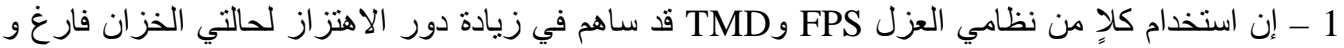

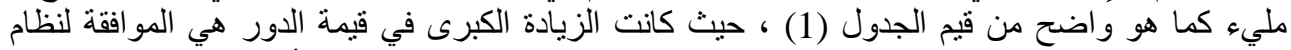

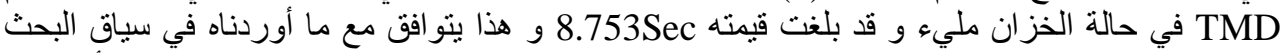

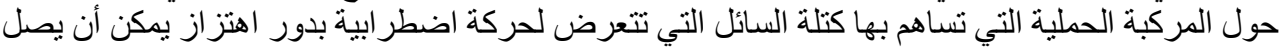
$10 \mathrm{Sec}$ 2 ـ أدى استخدام نظام العزل FPS إلى زيادة قيم الإزاحات المطلقة عند قمة الخزان لكن ما يعنينا في الدراسة

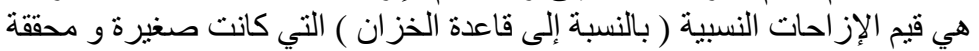

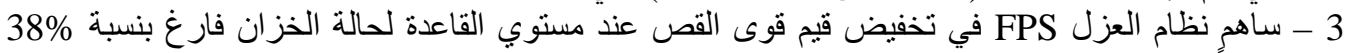

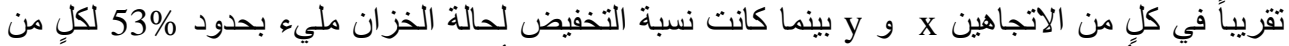

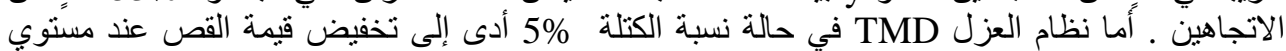

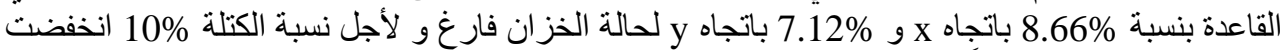

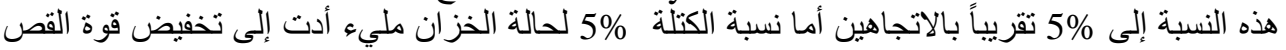

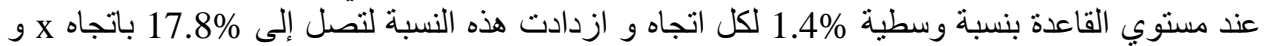
y 2.13\%

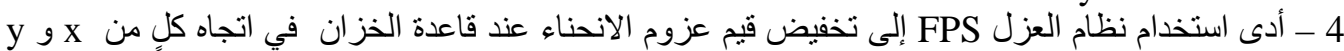

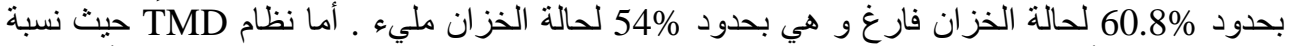

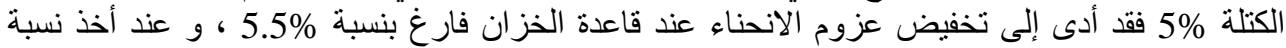

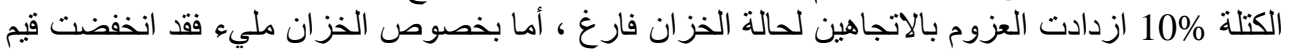

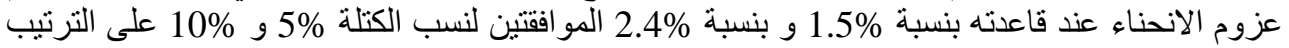

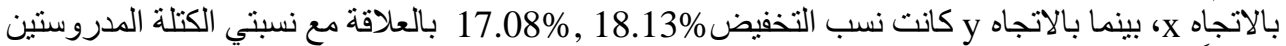

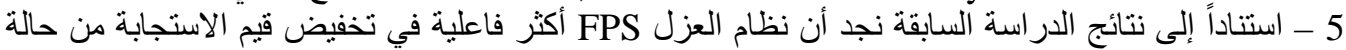

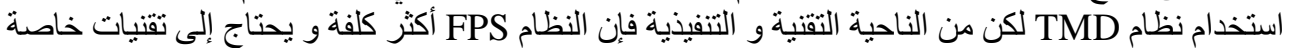

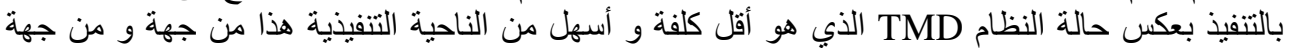

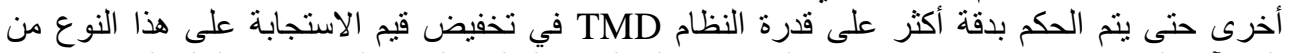

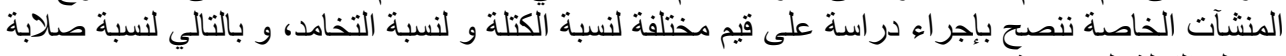

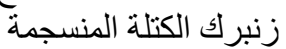

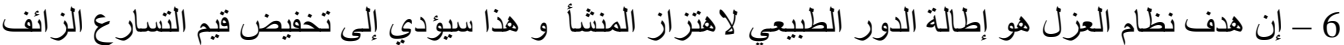

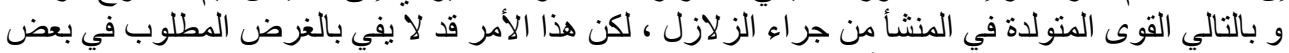

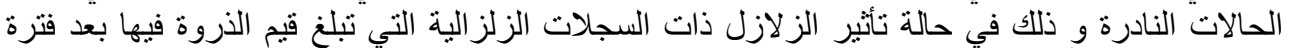

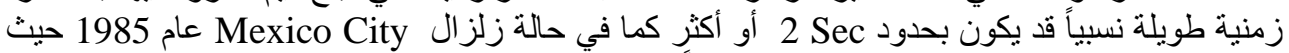

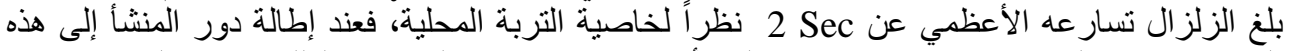

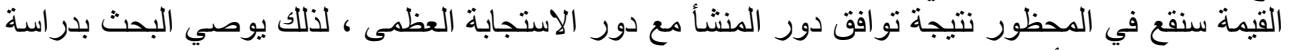

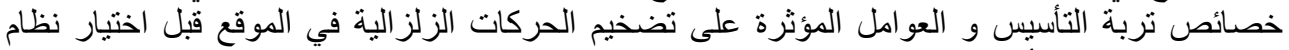

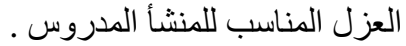

\section{المراجع العلمية}

1 - JAISWAL, O .R, RAI,D.C, JAIN,M.S. Review of Seismic Codes on LiquidContaining Tanks, Earthquake Spectra, Vol. 23,No.1,2007, 239-260

2- SAP 2000, Integrated Software for Structural Analysis \& Design Computers and Structures Inc, Berkeley, CA, 2004,790.

3 - LIVAOGLU, R, DOGANGUN, A. Simplified seismic analysis procedures for elevated tanks considering fluid-structure-soil interaction, Journal of Fluids and Structures, Vol.22, 2006, 421-439. 
4- LIVAOGLU, R. Investigation of seismic behavior of fluid-rectangular tanksoil foundation systems in frequency domain, Journal of Soil Dynamics and Earthquake Engineering, Vol. 28, 2008, 132-146.

5 - ASCE 7 - Minimum Design Load for Building and Other Structures, American Society of Civil Engineers, Reston, Virginia, USA, 2005, 560.

6 - JAISWAL, O.R, JAIN, S.K. Guidelines for Seismic Design of Liquid Storage Tanks, Indian Institute of Technology Kanpur, India, Report No IITK-GSDMAEQ-08,2007

7 -RAI, D.C. Seismic retrofitting of $R / C$ shaft support of elevated tanks, Earthquake Spectra. Vol.18, 2002, 745-760.

8 - ACI 371R-98, American Concrete Institute, Guide to the Analysis Design and Construction of Concrete-pedestal Water Tower, 1995, 345.

9 - SEZEN, H, LIVAOGLU, R, Dynamic analysis and seismic performance evaluation of above-ground liquid-containing tanks, Engineering Structures, Vol.30,2008, 794-803.

10 - EUROCODE -8 -Design of Structures for Earthquake Resistance, Part 4, Silos , Tanks, and Pipelines ,Final PT Draft .European Committee for Standardization, 2003.

11 - MALHOTRA, P.K., WENK,T.,WEILAND, M. Simple procedure of seismic analysis of liquid-storage tanks, Journal of Structural Engineering International ,IABSE, Vol..10, Nº. 3 , 2000, 197-201.

12-Earthquake Protection Systems (EPS), Friction Pendulum Seismic Isolation Bearing, 2008, 15 October, 2008.

<http://www.earthquake protection.com>.

13 - BARROSO,L. R. Performance Evaluation of Vibration Controlled Steel structures Under Seismic Loading, 2006, 15 March, 2009,. http://ceprofs.tamu.edu/ibarroso/research/papers/THESIS.PDF

14 -PRANESH, M., SINHA, R. VFPI: an isolation device for a seismic design, Journal of Earthquake Engineering And Structural Dynamics, U.S.A.Vol.29, Nº. 5, 2000, 603-627.

15 - FEMA 451, Instructional Material Complementing, Design Examples, 2006

16 - LIEDES, T. Improving the Performance of the Semi-Active Tuned Mass Damper, Academic dissertation ,Faculty of Technology of the University .of Oulu,2009, 150.

17 -CHEY, M .H., CARR, A .J., CHASE, J.G .Resetable Tuned Mass Damper and its application to isolated stories building system,14th World Conference on Earthquake Engineering, Beijing, China , 2008, 420-428.

18 - CHEY, M .H., Carr, A .J., Chase, J.G .Design of Semi-Active Tuned Mass Damper Building Systems using Resetable Devices, 8th Pacific Conference on Earthquake Engineering ‘Singapore, 2006, 351-360.

19- JULIO, C .M .On tuned mass dampers for reducing the seismic response of structures, Earthquake Engineering and Structural Dynamics ‘Vol. 34, 2005, 847865

20 - PAPOF, N.N, ZABERAEF, A.F. - Computation and Designing of the Concrete and Masonry Structures, SSSR Moscow, 1989, 400. 